\title{
AGGREGATION AND INTERMEDIATE PHASES IN DILUTE SPIN SYSTEMS
}

\author{
L. CHAYes \\ Department of Mathematics \\ $U C L A$
}

\author{
R. KoTECKÝ \\ Center for Theoretical Study \\ Charles University, Prague
}

and

S.B. SHLOSMAN

Department of Mathematics, UCI

\begin{abstract}
.
We study a variety of dilute annealed lattice spin systems. For site diluted problems with many internal spin states, we uncover a new phase characterized by the occupation and vacancy of staggered sublattices. In cases where the uniform system has a low temperature phase, the staggered states represent an intermediate phase. Furthermore, in many of these cases, we show that (at least part of) the phase boundary separating the low-temperature and staggered phases is a line of phase coexistence - i.e. the transition is first order. We also study the phenomenon of aggregation (phase separation) in bond diluted models. Such transitions are known, trivially, to occur in the large- $q$ Potts models. However, it turns out that phase separation is typical in bond diluted spin systems with many internal states. (In particular, a bond aggregation transition is not tied to a discontinuous transition in the uniform system.) Along the portions of the phase boundary where any of these phenomena occur, the prospects for a Fisher renormalization effect are deemed to be highly unlikely or are ruled out altogether.
\end{abstract}

Key words and phrases. Intermediate phases, annealed dilute systems, antiferromagnetic order, Potts models, aggregation, phase separation.

Partly supported by the NSF grant DMS-93-02023 (L.C.), the grants GAČR 202/93/0449 and GAUK 376 (R.K.), and NSF grant 9208029 (S.B.S.). 


\section{Introduction}

\section{Annealed Dilute Systems.}

Annealed dilute spin systems have, traditionally, received far less attention than their quenched counterparts: From the physical perspective, it is generally agreed that the experimental realizations of dilute spin systems are better described in the quenched approximation and, from the theoretical perspective, it is generally believed that the annealed-dilute problems are not substantially different from their uniform counterparts. Although we will not be discussing the applicability of annealed-dilute spin models, let us briefly address the first issue by noting that there are a host of systems - such as alloys or multi-component fluids - that are also described by dilute spin models. In many of these cases, it can be argued that the annealed version is the appropriate choice.

Let us turn attention to the second issue. According to the standard notions of universality, the nature of a phase transition should depend on only a limited number of details of the model. Thus, if we consider a typical lattice spin system described by the (formal) Hamiltonian

$$
H=-\sum_{i, j \in \mathbb{L}} J_{i, j}\left(\sigma_{i}, \sigma_{j}\right) .
$$

(where for simplicity we have restricted attention to pair interactions) the "important details" are presumed to be the dimension of the lattice $\mathbb{L}$, the range of the interaction and the general features (e.g. symmetries) of the spin variables $\sigma_{i}$ that are respected by the functions $J_{i, j}(-,-)$. The bond and site annealed versions of the Hamiltonian in the equation (1.1) are given by

$$
H_{b}=-\sum_{i, j \in \mathbb{L}} n_{i, j}\left(J_{i, j}\left(\sigma_{i}, \sigma_{j}\right)+\lambda_{i, j}\right)
$$

and

$$
H_{s}=-\sum_{i, j \in \mathbb{L}} n_{i} n_{j} J_{i, j}\left(\sigma_{i}, \sigma_{j}\right)-\mu \sum_{i \in \mathbb{L}} n_{i}+K_{s},
$$

respectively. In the equation (1.2a), $n_{i, j}$ is 0 or 1 indicating the presence or absence of a bond and it may be presumed, without loss of generality that for those pairs which are beyond the range of the interaction $\left(J_{i, j}\left(\sigma_{i}, \sigma_{j}\right) \equiv 0\right) n_{i, j}$ is always zero. In the equation (1.2b), $n_{i}$ is similarly either 0 or 1 and $K_{s} \equiv K_{s}\left[\left(n_{i}\right)\right]$ represents possible additional terms involving the $\left(n_{i}\right)$ alone. $^{1}$

The partition function for the systems described by the equations (1.2) are defined by summing $e^{-\beta H}$ over all bond/site configurations and tracing out the spin

\footnotetext{
${ }^{1} \mathrm{~A}$ bond-bond interaction term can also be added to the Hamiltonian in (1.2a) but we regard this as an unnecessary complication. As such, the above described bond-diluted models are usually referred to as uncorrelated. However, it is clear that as soon as the spin interactions are taken into account, (i.e. after the annealed trace is performed) there will, in general, be correlations among the bond variables. In both the bond and site diluted cases, we will take the minimum value of $J_{i, j}\left(\sigma_{i}, \sigma_{j}\right)$ to be zero. In the bond-diluted models this convention is implemented without loss of generality since the difference can be absorbed into the $\lambda_{i, j}$. However, in the case of site-dilution, this convention is not without loss of generality and this is the principle reason for the extra term
} 
degrees of freedom according to a pre-specified spin-space measure (which may include magnetic field type terms not written into the equations (1.2)). One can conceive of being able to explicitly perform these operations in exactly this order and, after the first step has been achieved, ending up with an effective uniform system Hamiltonian. As such, it is difficult to believe that the "essential features" of the uniform and dilute system differ in any dramatic way. Therefore it is anticipated that the phase structure and phase transitions will be of the same type with or without the annealed dilution.

Let us pause to illustrate this procedure for the annealed bond-dilute problems. The partition function (on some suitably finite $\mathbb{L}$ ) can be written as

$$
\begin{aligned}
\mathbb{Z}_{\mathbb{L}}=\int d^{\mathbb{L}} \sigma \sum_{n_{i j}} \prod_{i, j \in \mathbb{L}} e^{-\beta\left[n_{i j} J_{i, j}\left(\sigma_{i}, \sigma_{j}\right)-\lambda_{i, j} n_{i j}\right]} \propto \\
\propto \int d^{\mathbb{L}} \sigma \prod_{i, j \in \mathbb{L}}\left[p_{i j} e^{-\beta J_{i, j}\left(\sigma_{i}, \sigma_{j}\right)}+\left(1-p_{i, j}\right)\right],
\end{aligned}
$$

where $\frac{p_{i, j}}{1-p_{i, j}}=e^{\beta \lambda_{i, j}}$. Thus, in one stroke, we have produced a uniform system Hamiltonian of the form in the equation (1.1):

$$
\tilde{H}=-\sum_{i, j \in \mathbb{L}} \tilde{J}_{i, j}\left(\sigma_{i}, \sigma_{j}\right)
$$

at some inverse temperature $\tilde{\beta}$ where the new $\tilde{\beta}$ and $\tilde{J}_{i, j}$ are given in terms of the old by the relations

$$
e^{-\tilde{\beta} \tilde{J}_{i, j}\left(\sigma_{i}, \sigma_{j}\right)}=p_{i, j} e^{-\beta J_{i, j}\left(\sigma_{i}, \sigma_{j}\right)}+1-p_{i, j}
$$

In particular, when the $J_{i, j}$ can only take on two values - as is the case in the Potts models - all that has changed is the temperature.

Over the years, these sorts of conclusions have been bolstered by a myriad of other exact results (e.g. [SM], [EG] and [ST], see [St] and references therein) along with some additional considerations (see $[\mathrm{F}]$ ). All leaves us with the quiescent picture of a general stability to annealed types of disorder and no real need to study these models as separate entities in their own right.

\section{Fisher Renormalization and Phase Transitions in Annealed Systems.}

Against the above mentioned background, in the late 1960's, Fisher addressed the problem of continuous transitions in systems with "hidden" constraints $[\mathrm{F}]$. An example of such a system is described by the equations (1.2) in a constrained ensemble defined by a fixed concentration of bonds or sites. Now according to the likes of the equations (1.4), the bond concentration is essentially the same as the energy density. It therefore follows that when a critical phase boundary (in the extended parameter space) is approached at any finite angle, the concentration remains approximately constant provided that the specific heat exponent, $\alpha$, is negative. However, if $\alpha>0$, it becomes necessary to devise a drastic line of approach in order to stay at fixed concentration. This in turn implies that when a 
the so called Fisher renormalization effect which means that nothing changes if $\alpha$ is negative but if $\alpha>0$,

$$
\begin{gathered}
\alpha \longrightarrow \alpha^{\star}=\frac{-\alpha}{1-\alpha}, \\
b \longrightarrow b^{\star}=\frac{b}{1-\alpha} \quad b=\beta, \gamma, \nu,
\end{gathered}
$$

and

$$
c \longrightarrow c^{\star}=c \quad c=\delta, \eta .
$$

The arguments in $[\mathrm{F}]$ are straightforward, essentially rigorous and, for several Ising type systems, actually provide a more satisfactory account of the experimental data than the uniform exponents. Of course the equations (1.5) rely crucially on the supposition - Hypothesis $B$ in $[\mathrm{F}]$ - that the phase structure and the phase transitions of the pure system have not been corrupted by the addition of the dilution degrees of freedom.

In this paper, we provide certain evidence to the contrary. In particular we show that the global results suggested by the exact solutions are exceptional situations and that typically the extended phase diagram is beset with first order transitions and intermediate phases. The foremost of our results are:

- the existence, for site-diluted models, of an intermediate phase characterized by the occupation and vacancy of staggered sublattices and

- the proof of a discontinuous bond aggregation transition for bond diluted models.

Minimal hypotheses are required to establish the above effects. The basic ingredients are [i] many internal states, [ii] a mild restriction on the degeneracy of the lowest energy spin-states and, [iii] (when relevant) a condition that ensures that the low temperature behavior of the uniform system is not excessively frustrated. However, for ease of exposition, in this work we confine attention to reflection positive models. This is by no means a requirement. Indeed, in more general annealed dilute systems, one finds a myriad of additional phase transitions of this sort. [CKS]

The above phenomena are entropy driven and thus, by in large, have escaped notice. For example, it appears that the staggered phase eluded the renormalization group analysis of the site diluted Potts model presented in [NBRS]. (See, however, [RL].) On the other hand, site aggregation in annealed site-diluted models is energy driven and, as such, has been well understood for some time. Nevertheless, to our knowledge, this transition has not been studied by rigorous methods. In this paper, we will also provide

- a proof of a discontinuous site aggregation transition for site-diluted models.

Clearly, on the portion of the phase boundary corresponding to a discontinuous transition, the Fisher effect is ruled out. Further, when the phase transition is an entrance to or an exit from an intermediate phase, one is bound to be suspicious. To underscore this point we prove, in a number of cases, that at least part of the phase boundary between the staggered phase and the low temperature phase is also discontinuous.

Of course, nothing in this paper proves that a Fisher renormalization scheme is impossible. In the first place, whenever the transition (in the extended parameter space) is continuous, the arguments of Fisher still apply. However, it might happen that the $\alpha, \ldots, \eta$ on the right hand side of the equations (1.5) do not correspond 
plausible that at weak dilution, Hypothesis $B$ is still in effect. Thus, if part of the phase boundary is first order, we can envision a critical line of the uniform type of transition emanating from the uniform critical point and joining up with the discontinuous portion of the transition line at a tricritical point. However, without additional detailed arguments, it is equally plausible that the phase boundary in the vicinity of the uniform system is a weakly first order line.

Let us now discuss the physical origins of these phenomena.

\section{Aggregation and Staggered Phases: Heuristics of the Transitions.}

Once they are spelled out, the underlying reasons for these effects are not particularly difficult to understand. Let us start with the staggered phases. It will be sufficient, for present purposes, to consider the nearest neighbor Potts Hamiltonian on $\mathbb{Z}^{d}$. Thus in the equation (1.2a), we take $\sigma_{i} \in\{1,2, \ldots, q\}, J_{i, j}=J\left(\delta_{\sigma_{i}, \sigma_{j}}-1\right)$ for $|i-j|=1$ and zero otherwise and, to keep things simple, set $K_{s}=0$. Consider the case where $e^{\beta J}$ is large, $q$ is large and $e^{\beta \mu}$ is small. Then the partition function (or activity) of an isolated particle is $q e^{\beta \mu}$ which we will now regard as appreciably - but not enormously - large. Now consider the situation when two particles are neighbors: they must either reside in the same state, which restricts the pair to a small fraction, $1 / q$, of the states that they had in isolation or suffer an severe energetic penalty of $e^{-\beta J}$ if they choose to disagree. Thus there is a strong effective repulsion between neighbors and the system is reminiscent of a hard squares problem. For hard squares, it is known [D], [FLIS III] that a pair of staggered states exists at some fairly reasonable value of the activity. Thus, the $\beta J=-\beta \mu=q=\infty$ limit of this problem is understood and, in a certain sense, all that remains is to show that this situation is stable enough to persist at finite temperatures.

In this context, it is worth noting that a restricted version of this problem was analyzed some time ago in $[\mathrm{RL}]$. There, the system considered was the lattice version of the Widom-Rowlinson model which may be formally identified with the sitediluted Potts model at $J=+\infty$. In [RL], the existence of staggered phases was indeed demonstrated using, more or less, the above line of reasoning. However, the generality of this phase and its importance in the context of dilute systems was not discussed.

Let us now turn attention to the problem of site aggregation. We will be extremely brief because the heuristics are adequately described elsewhere, e.g. [St]. Consider, for example, the above model with the additional term $K_{s}=$ $-\kappa \sum_{|i-j|=1} n_{i} n_{j}$ where $\kappa>0$. Let us now envision the constrained ensemble at zero temperature with a positive fraction of sites and vacancies: It is clear that energetics will push all the sites together into one big cluster which is an act of phase separation in its purest form. All things considered, it is not difficult to show that this general situation persists at finite temperatures.

Finally, we come to the question of bond aggregation. Here, in the context of the constrained ensemble it has been argued that there will not be phase separation at zero temperature because "there is no energetic advantage in that". This, however, is a little naive since, in a constrained ensemble, there are entropic effects even at $T=0$. As for our discussion, let us go back to the equations (1.4) and consider, e.g. for an isotropic nearest neighbor model, the limit $\beta \rightarrow \infty$ with $p=e^{\beta \lambda} /\left(1+e^{\beta \lambda}\right)$ fixed. It has already been discussed that if the original model is a Potts model then (even at $T=0$ ) we still have a Potts model at a finite effective temperature. As 
to aggregation transitions in the diluted versions.

It seems, therefore, that an aggregation transition is tied to a first order transition in the uniform system - and this appears to be the current accepted wisdom. However, this reasoning has no basis and the answer turns out to be far simpler: Consider, for example, any $q$-state ferromagnetic model. By this we mean that $\sigma_{i}$ has $q$ states and $J_{i, j}\left(\sigma_{i}, \sigma_{j}\right)=0$ if $\sigma_{i}=\sigma_{j}$ and is positive otherwise. Under the same limit $(\beta \rightarrow \infty$ with the $p$ 's fixed) we arrive at: The $q$-state Potts Model. C.f. the equation (1.4b). Thus, under quite general circumstances $-q$ large, and, say, nearest neighbor interactions, we find that the transition at zero temperature is first order. Under these conditions, it is again not terribly difficult to show that this situation persists at finite temperature.

\section{Strategic Overview, Organization and Summary of Results.}

The strategy that is throughout this work is the standard approach in theory of phase transitions: the method of contours. We start by focusing attention on the smallest possible subsystem that is capable of exhibiting the characteristics of the phase in question. Most often, this will be an hypercube of side 2. For example, a staggered phase is exhibited by the corresponding checkered pattern of the occupation variables on the hypercube or a low density phase is exhibited by a hypercube that is devoid of sites.

In the case of a single phase, once the phase signature has been defined, any hypercube that is not of this type is considered a contour (or part of a contour). The existence of the phase is established by a demonstration that contours are "rare".

In the case of a region of coexisting phases (e.g. the staggered phases) there are different possible modes of correct behavior on the elementary hypercubes. These should be a priori of equal probability. Again, any hypercube not exhibiting one of the characteristic behaviors belongs to a contour. Moreover, to prove that the phases coexist, it must be shown that the simultaneous presence of two or more types of hypercubes is improbable. This amounts to showing that, under such circumstances, contours are present and the proof again reduces to showing that contours are rare.

Finally, in cases without underlying symmetry, we allow the probabilities to depend on a variable parameter such as the temperature or chemical potential. Two things must be established: (a) throughout the range of parameter, contours must be suppressed and (b) in the two extreme regions of the range of this parameter, different phases dominate. From this it follows that there is a point of phase coexistence and furthermore (since the contours are extremely rare) the probabilities of the individual behaviors take a jump.

In this paper, the above program is implemented by the methods of reflection positivity. These methods allow us to estimate the various local probabilities in terms of the partition function where the global configuration takes on the appropriate characteristic in every hypercube. In addition, these methods allow us to estimate the probability of a contour as the product of factors - the number of which scales with the size of the contour. These factors can then be evaluated in terms of the probability that the entire system consists of a single contour.

The principal price of using the RP methods is that we must limit, severely, the class of models that we wish to study. In addition, we must be content with a 
diluted ferromagnets where the high temperature, low temperature and staggered phases meet. In future papers we will study systems with more general interactions, sometimes using the methods of Pirogov and Sinai. In the general situation, it turns out that a plethora of additional staggered phases are possible. Furthermore, we will demonstrate the existence of all the phases and phase transitions discussed here for systems with continuous spins, such as the $X Y$ or Heisenberg models.

For the staggered phases and their generalizations, the ratio of output to effort is approximately the same for the RP versus PS methods. However, in the case of the bond aggregation transitions, the RP methods provide a reliable technique that allows a reasonably general proof at little cost. By contrast, the contour methods would require a difficult construction along the lines of [MS] to prove these results.

The organization of this paper is as follows:

Section 2 will be completely devoted to the analysis of the two-dimensional sitediluted Potts model. For this case, we have pushed hard on our methods to obtain nearly complete results. The phase diagram for this system is illustrated in Figure 2.1 and the principal result of this section, Theorem 2.1. is a proof of the major part of this picture. Section 2 is pretty much a self-contained piece of work. Most of the technical results (and a good deal of the notation) used in the rest of the paper will be developed in this context.

In Section 3, we analyze the problem of staggered ordering in a more general setting and prove the relevant parts of Theorem 2.1 for an extended class of large $q$ nearest neighbor models (Theorem 3.1).

In Section 4, we treat the problem of (first order) aggregation transition for the bond- and site-diluted models. Under suitable hypothesis we show that these transitions occur: in the bond-diluted case, large $q$ is required. However, in the site-diluted case, these transitions occur with only minimal hypotheses. 


\section{Phase diagram of the $2 d$ site-Diluted Potts Model}

\section{Definitions and Statement of Results.}

Our starting point will be to define the $q$-state Potts Hamiltonians on $\mathbb{Z}^{2}$. Beginning with a finite volume system, we will consider our models on the 2-dimensional tori that are given by

$$
\mathcal{T}_{N}=\left\{i \in \mathbb{Z}^{2} \mid 0 \leq i_{k} \leq N ; k=1,2\right\}
$$

together with the formal identifications $\left(N, i_{2}\right)=\left(0, i_{2}\right)$ and $\left(i_{1}, N\right)=\left(i_{1}, 0\right)$. Here, and throughout this paper, we will assume that $N$ is of the form $2^{k}$. If $i, j \in \mathcal{T}_{N}, i$ and $j$ are deemed to be neighbors if one of their coordinates agree $(\bmod N)$ and the other differs $(\bmod N)$ by 1 . When a pair of points, $i$ and $j$ satisfies this criterion, we use the notation $\langle i, j\rangle .{ }^{2}$ With this in mind, the site-diluted Potts Hamiltonian on the torus $\mathcal{T}_{N}$, is given by

$$
H_{N}\left(n_{N}, \sigma_{N}\right)=-J \sum_{\langle i, j\rangle} n_{i} n_{j}\left(\delta_{\sigma_{i}, \sigma_{j}}-1\right)-\mu \sum_{i} n_{i}-\kappa \sum_{\langle i, j\rangle} n_{i} n_{j}
$$

where the first and third terms run over all neighboring pairs of $\mathcal{T}_{N}, n_{i}=0$ or 1 indicates the absence or presence of a particle at the site $i \in \mathcal{T}_{N}$, the $\sigma_{i}$ 's denote the usual $q$-state Potts variables, $\sigma_{i} \in\{1, \ldots, q\}$ and $\delta_{\sigma_{i}, \sigma_{j}}=1$ if $\sigma_{i}=\sigma_{j}$ and is zero otherwise. The partition function $\mathcal{Z}_{N, \beta}=\mathcal{Z}_{N, \beta}(\mu, \kappa, J)$ is given by the annealed trace

$$
\mathcal{Z}_{N, \beta}=\sum_{\substack{n_{i}=0,1 \\ i \in \mathcal{T}_{N}}} \sum_{\substack{\sigma_{i} \in\{1, \ldots, q\} \\ i \in \mathcal{T}_{N}, n_{i}=1}} \exp \left\{-\beta H_{N}\left(n_{N}, \sigma_{N}\right)\right\}
$$

As usual, the partition function serves as the normalization constant for the finite volume Gibbs states, $\langle-\rangle_{N, \beta}^{J, \mu, \kappa}$, that assigns to the configuration $\left(n_{N}, \sigma_{N}\right)$ a weight proportional to $\exp \left\{-\beta H_{N}\left(n_{N}, \sigma_{N}\right)\right\}$. Therefore, the physical interpretation of the restricted sum over spin configurations in the equation (2.3) is that the spins $\sigma_{i}$ are simply not present unless $n_{i}=1$. Alternatively, we may stipulate that the spin variables are always present - and should be summed over — while the $n_{i}$ represent additional degrees of freedom that mediate the interaction. It is easily seen that the latter problem is equivalent to the former after a shift in $\mu$ by the amount $\frac{1}{\beta} \log q$. In this paper, we keep with the original perspective.

Remark. We will assume throughout our discussion of the Potts models that $J>0$ and $\kappa \geq 0$. If $J \leq 0$ and $\kappa>0$, the staggered phases probably do not occur for any value of $\beta$ or $\mu$. If $J>0$ and $\kappa<0$, the interaction between neighboring particles is a priori repulsive and the existence of the staggered phases comes as no real surprise. In fact, the Ising $(q=2)$ version of this case was investigated in the guise of the Blume-Emery Griffiths model [HB]. (Albeit with non-rigorous methods.) Not surprisingly, it was concluded that for $\kappa$ negative and below a certain value, a staggered phase emerges. The problems with $J>0$ and $\kappa<0$ could easily be incorporated into the forthcoming, but this would require a proviso following each formula. Hence, although this region is both, in principle and in

${ }^{2}$ Despite the comma, $\langle i, j\rangle$ is not an ordered pair. Thus we may identify the neighboring pair 
practice, more straightforward than the region $J>0$ and $\kappa>0$, we will postpone its treatment until we get to the general $q$-state problems in the next section. In fact, once we allow $\kappa<0$, we can even prove the existence of staggered phases for weakly antiferromagnetic $(J<0)$ interactions. However, these problems are not of sufficient interest to warrant a separate treatment in their own right.

FIGURE 2.1

Let us summarize our claims concerning the phase diagram of the diluted $q$ state Potts model defined by the equations (2.2) and (2.3). For some fixed values of $\kappa \in(0, J)$ and $q \gg 1$, the phase diagram that will emerge from our analysis is schematically shown in Figure 2.1. (We have, of course, allowed ourselves some artistic leeway.) In a region around the point $(\mu=\infty, \beta=\infty), q$ different ordered phases coexist, while on the other hand, if $-\mu$ is large and/or $\beta$ is small, there is a unique "disordered" phase. Close to the axes $\mu=\infty$ and $\beta=\infty$, the disordered phases and the low temperature phases meet directly. Further away from the axes, the staggered phases are sandwiched between the extremes. These new phases are characterized by the preferential occupation of the even or odd sublattices and zero spontaneous magnetization. Much of the boundary between this region and the region of $q$ ordered phases is also the line of first order transitions at which $q+2$ phases coexist. On the other hand, the boundary between this region and the high temperature phase is conceivably a line of continuous transitions. In our analysis, we will investigate the behavior in four (partially overlapping) regions where we 
in these regions always involve a transition into the ordered state; unfortunately, we have no control of the phase diagram along the curve where the disordered and staggered phases meet - in particular, at its two ending triple points.

In the forthcoming, a given state, "*", corresponding to parameters $\kappa, \beta$, and $\mu$, will be denoted by \langle\rangle$_{\kappa, \beta, \mu}^{(*)}$ (sometimes omitting various parameters). As is the case of the standard Potts model, the ordered phase can be characterized by a significant probability $\left\langle\delta_{\sigma_{i}, m}\right\rangle^{(m)}$ that a given spin attains a fixed value $m$. Furthermore, the average, $\left\langle\chi_{b}^{(m)}\right\rangle^{(m)}$, of the indicator for the event that a given bond $b$ has both its endpoints occupied and in the spin state $m$ attains an appreciable value in this phase. To describe the staggered phases we consider elementary squares

$$
c=c(j)=\left\{i \in \mathcal{T}_{N} \mid j_{k} \leq i_{k} \leq j_{k}+1, k=1,2\right\}
$$

Let $I_{A}(c)$ denote the event that all the even sites of $c$ are occupied and all the odd sites of $c$ are vacant:

$$
I_{A}(c)=\left\{n_{N}, \sigma_{N} \mid n_{i}=1 ; i \in c, i_{1}+i_{2} \text { even }, n_{i}=0 ; i \in c, i_{1}+i_{2} \text { odd }\right\} .
$$

To define $I_{B}(c)$, we exchange of the roles of the even and odd sites in the equation (2.5). The phases, to be denoted \langle\rangle$^{(\mathrm{A})}$ and \langle\rangle$^{(\mathrm{B})}$, will be characterized by a large probability of the events $I_{A}$ and $I_{B}$, respectively. We use $\chi_{A}(c)$ and $\chi_{B}(c)$ to denote the indicator functions of the events $I_{A}(c)$ and $I_{B}(c)$. Finally, the characterization of the unique "high temperature-low density" disordered phase depends on the region: in one region it is characterized by a high probability $\left\langle\chi_{b}^{\text {(dis) }}\right\rangle^{(\mathrm{d})}$ that a bond is occupied by different spins, $n_{i}=n_{j}=1, \sigma_{i} \neq \sigma_{j}$, while in another it is characterized by a low density, $\left\langle\delta_{n_{i}, 1}\right\rangle^{(\mathrm{d})}$.

Our claims about the phase diagram can now be formulated as the following statements concerning the existence of distinct infinite volume Gibbs states ${ }^{3}$ corresponding to the given values of parameters $\kappa, \mu$, and $\beta$.

\section{Theorem 2.1.}

Consider the site diluted q-state Potts models with $J$ and $\kappa$ fixed and satisfying $0<\kappa<J$ and suppose that $q$ is (sufficiently) large and fixed. Then there are regions $R_{I}=R_{I}^{o} \cup R_{I}^{d}, R_{I I}=R_{I I}^{o} \cup R_{I I}^{d}$, and $R_{I I I}=R_{I I I}^{o} \cup R_{I I I}^{S}$ (the regions $R_{I}, R_{I I}$, and $R_{I I I}$ overlap) such that $R_{I}^{o} \cap R_{I}^{d}=\gamma_{I}, R_{I I}=R_{I I}^{o} \cap R_{I I}^{d}=\gamma_{I I}$, and $R_{I I I}^{o} \cap R_{I I I}^{S}=\gamma_{I I I}$ are continuous curves. Moreover, there is a "small" number $\epsilon$ such that:

i) The region $R_{I}^{o}$ is defined by the existence of $q$ different states \langle\rangle$_{\kappa, \beta, \mu}^{(m)}, m=$ $1, \ldots, q$, for which

$$
\left\langle\delta_{\sigma_{i}, m}\right\rangle_{\kappa, \beta, \mu}^{(m)} \geq 1-\epsilon
$$

while the region $R_{I}^{d}$ is defined by the existence of a disordered state \langle\rangle$_{\kappa, \beta, \mu}^{(\mathrm{d})}$ for which

$$
\left\langle\chi_{b}^{(\mathrm{dis})}\right\rangle_{\kappa, \beta, \mu}^{(\mathrm{d})} \geq 1-\epsilon .
$$

\footnotetext{
${ }^{3}$ For the various values of the parameters, we only prove that at least the states that are characterized in Theorem 2.1 exist. In principle, this does not exclude the existence of some additional phases. However, with a bit more work this could be achieved using Pirogov Sinai
} 
ii) The region $R_{I I}^{o}$ is characterized by the existence of ordered states as described in item (i) above. The region $R_{I I}^{d}$ is defined by the existence of a disordered state for which

$$
\left\langle\delta_{n_{i}, 1}\right\rangle_{\kappa, \beta, \mu}^{(\mathrm{d})} \leq \epsilon .
$$

iii) In the region $R_{I I I}^{o}$ there are ordered states as described above. In $R_{I I I}^{S}$ there are two states \langle\rangle$_{\kappa, \beta, \mu}^{(\mathrm{A})}$ and \langle\rangle$_{\kappa, \beta, \mu}^{(\mathrm{B})}$ for which

$$
\left\langle\chi_{A}(c)\right\rangle_{\kappa, \beta, \mu}^{(\mathrm{A})} \geq 1-\epsilon
$$

and

$$
\left\langle\chi_{B}(c)\right\rangle_{\kappa, \beta, \mu}^{(\mathrm{B})} \geq 1-\epsilon
$$

respectively.

In (i)-(iii) above, both characteristic behaviors of the regions $R_{I}-R_{I I I}$ are found at all points of the curves $\gamma_{I^{-}} \gamma_{I I I}$. In short, these are curves of phase coexistence; explicitly, on $\gamma_{I}$ and $\gamma_{I I}$ there coexist $q+1$ phases and on $\gamma_{I I I}$ there coexist $q+2$ phases. Further, the curves $\gamma_{I^{-}} \gamma_{I I I}$ can be represented as (graphs of) continuous functions.

iv) Finally, there is a region $R_{I V}$ in which there is a unique Gibbs state satisfying the conditions of complete analyticity.

For convenience, the above regions and curves are illustrated in Fig. 2.2. 


\section{Reflection Positivity and Other Tools.}

Our analysis in this work relays heavily on the fact that all the systems we consider are reflection positive (RP). In the discussion on reflection positivity that is to follow, we will be as terse as possible. Indeed, we will supply just enough information to define our notation. For more details, the reader is urged to consult the original references [FSS], [FL], [FILS I], or the review article [S].

In order to permit the unimpeded use of these results in later sections, we must work in a slightly more general context: in particular $\mathcal{T}_{N}$ will now denote the $d$ dimensional torus of linear scale $N$ and the spin variables will belong to an arbitrary (but in this paper discrete) space.

We will also allow for the possibility of dynamical variables on the bonds: Bonds are defined according to the obvious generalization of the previous discussion; a pair of sites with $d-1$ of their coordinates in agreement and one of their coordinates differing by exactly one unit constitutes a bond. We will denote the set of bonds of $\mathcal{T}_{N}$ by $\mathcal{B}_{N}$.

Let $P$ denote a generic "hyperplane of sites" for the torus $\mathcal{T}_{N}$. By way of example, we may consider

$$
P_{0}=\left\{i \in \mathcal{T}_{N} \mid i_{1}=0 \text { or } i_{1}=\frac{N}{2}\right\}
$$

In this work we will consider only planes $P$ which contain sites and are orthogonal to one of the coordinate axis. Let $P^{+}$and $P^{-}$denote the corresponding "right" and "left" halves of the torus, e.g.

$$
P_{0}^{+}=\left\{i \in \mathcal{T}_{N} \mid 0 \leq i_{1} \leq \frac{N}{2}\right\}
$$

If $i \in \mathcal{T}_{N}$, let $\vartheta_{P}(i) \in \mathcal{T}_{N}$ denote the image site of $i$ reflected by the hyperplane $P$ and, in general, if $\left\{i^{(1)}, \ldots i^{(k)}\right\} \subset \mathcal{T}_{N}$, let $\vartheta_{P}\left(\left\{i^{(1)}, \ldots i^{(k)}\right\}\right)=\left\{\vartheta_{P}\left(i^{(1)}\right), \ldots \vartheta_{P}\left(i^{(k)}\right)\right\}$. Let $\Sigma$ denote the spin space for the spin variables at the sites $i \in \mathcal{T}_{N}$ and let $\Xi$ denote the space of variables for the bonds $\langle i, j\rangle \in \mathcal{B}_{N}$. (For the site-diluted Potts model, we may take $\Sigma=\{0,1, \ldots, q\}$, 0 corresponding to $n_{i}=0$, and the values 1-q corresponding to $n_{i}=1$ and the appropriate value of $\sigma_{i}$. Here we would have $\Xi=\{1\}$ but for the bond-diluted models, we will have $\Xi=\{1,0\}$.)

Let $\left(S_{N}\right)$ be the notation for a spin configuration on $\mathcal{T}_{N}$ and let $S_{i}$ denote the value of the spin at the site $i$. Then we will use $\vartheta_{P} S_{i}$ to denote the value of the spin at the site $\vartheta_{P}(i)$. Similar notation applies to the bond variables: $\left(B_{N}\right)$ will be notation for a bond configuration, $B_{\langle i, j\rangle}$ will serve as notation for the individual values and we define $\vartheta_{P} B_{\langle i, j\rangle}=B_{\left\langle\vartheta_{P}(i), \vartheta_{P}(j)\right\rangle}$. Finally, if $f\left(S_{N} ; B_{N}\right)$ is a function that depends only on the configuration in $P^{+}: f=f\left(S_{i^{(1)}}, \ldots, S_{i(n)} ; B_{\langle i, j\rangle(1)}, \ldots, B_{\langle i, j\rangle}(m)\right)$ with $i^{(1)} \ldots i^{(n)}$ and $\langle i, j\rangle^{(1)}, \ldots\langle i, j\rangle^{(n)}$ in $P^{+}$we will say that $f \in \mathcal{F}_{N}^{P^{+}}$. Further, if $f \in \mathcal{F}_{N}^{P^{+}}$, we may define $\vartheta_{P} f$ (which, by analogy with the preceding notation would belong to $\left.\mathcal{F}_{N}^{P^{-}}\right)$by saying that for each $\left(S_{N} ; B_{N}\right)$,

$$
\begin{aligned}
& \vartheta_{P} f\left(S_{i^{(1)}}, \ldots, S_{i^{(m)}} ; B_{\langle i, j\rangle}(1), \ldots, B_{\langle i, j\rangle}(n)\right)= \\
& =f\left(\vartheta_{P} S_{i^{(1)}}, \ldots, \vartheta_{P} S_{i^{(m)}} ; \vartheta_{P} B_{\langle i, j\rangle}(1), \ldots, \vartheta_{P} B_{\langle i, j\rangle}(n)\right) .
\end{aligned}
$$

We may also, in a natural fashion, use $\vartheta_{P}$ to map $\mathcal{F}_{N}^{P^{-}} \rightarrow \mathcal{F}_{N}^{P^{+}}$and, in this sense, we have $\vartheta_{P}^{2}=1$. We shall omit any further explicit references to $\vartheta_{P}$ as a map from $\mathcal{F}_{N}^{P^{-}}$to $\mathcal{F}_{N}^{P^{+}}$since this would only serve to double the length of the various 
Definition. A state $\langle-\rangle$ on the set of configurations $\Sigma^{\mathcal{T}_{N}} \times \Xi^{\mathcal{B}_{N}}$ is said to be reflection positive, or reflection symmetric, with respect to the reflections $\vartheta_{P}$ if, for every $f \in \mathcal{F}_{N}^{P^{+}}$,

$$
\left\langle f \vartheta_{P} f\right\rangle \geq 0
$$

while for any $f, h \in \mathcal{F}_{N}^{P^{+}}$,

$$
\left\langle f \vartheta_{P} h\right\rangle=\left\langle h \vartheta_{P} f\right\rangle
$$

Proposition 2.2. Let $H_{N}\left(S_{N} ; B_{N}\right)$ denote a (Hamiltonian) function of the configurations on the torus $\mathcal{T}_{N}$ and let $\langle-\rangle_{N, \beta}$ denote the (Gibbs) state that assigns the weight proportional to $e^{-\beta H_{N}}$ to the configuration $\left(S_{N} ; B_{N}\right)$. Suppose that $H_{N}$ admits an expression of the form

$$
H_{N}=G_{N}+\vartheta_{P} G_{N}
$$

with $G_{N} \in \mathcal{F}_{N}^{P^{+}}$. Then the state $\langle-\rangle_{N, \beta}$ is reflection positive with respect to $\vartheta_{P}$.

Proof. This demonstrated in any of the references [FL], [FILS I] or [FSS]. See, e.g. [S] Theorem 2.1.

Let us consider the elementary hypercubes

$$
c=c(j)=\left\{i \in \mathcal{T}_{N} \mid j_{k} \leq i_{k} \leq j_{k}+1, k=1, \ldots, d\right\}
$$

and let $b$ denote a configuration (pattern) or a collection of configurations on the bonds and sites of the hypercube $c$. We use $I_{b}(c)$ as notation for the set of configurations $\left(S_{N} ; B_{N}\right)$ for which the restriction to $c$ displays this pattern and finally, $\chi_{b}(c)$ as the indicator for the event $I_{b}(c)$.

We may reflect the pattern $b$, repeatedly, through the various hyperplanes $P$ until the pattern covers the entire torus. Then, if $\Lambda$ is a collection of bonds and sites, we may consider the event $I_{b}(\Lambda)$ that $\left(S_{N}, B_{N}\right)$ restricted to $\Lambda$ displays this periodic extension of the pattern $b$. The indicator for the event $I_{b}(\Lambda)$ will be denoted by $\chi_{b}(\Lambda)$.

Our principal usage of reflection symmetry will be the so called chessboard estimate for contours:

Lemma 2.3. Let $\left\{c_{\ell}\right\}$ be a collection of distinct (but possibly overlapping) hypercubes and consider a particular behavioral pattern $b_{\ell}$ associated with each cube $c_{\ell}$. Then

$$
\left\langle\prod_{\ell} \chi_{b_{\ell}}\left(c_{\ell}\right)\right\rangle_{N, \beta}^{J, \mu, \kappa} \leq \prod_{\ell}\left(\left\langle\chi_{b_{\ell}}\left(\mathcal{T}_{N}\right)\right\rangle_{N, \beta}^{J, \mu, \kappa}\right)^{\frac{1}{\left|N^{d}\right|}} .
$$

Proof. The proof follows exactly the methods of [FL]; see Theorem 2.2 and the equations (1.42) and (1.44) in [FL].

The following result from $[\mathrm{KS}]$ is useful in conjunction with reflection positivity to establish the existence of discontinuous transitions:

Lemma 2.4. Let $a$ and $b$ denote two distinctive patterns on a cube $c \in \mathcal{T}_{N}$. Let $H$ be a Hamiltonian that depends on a control parameter, denoted by $\alpha$, that lies in the range $\left[\alpha_{a}, \alpha_{b}\right]$ and let $\langle-\rangle_{N, \alpha}$ denote the Gibbs state on $\mathcal{T}_{N}$ induced by the 
that $B \leq\left[\frac{1}{2}+\sqrt{\frac{1}{2}-\frac{A}{2}}^{2}\right.$ and let $\epsilon_{a}, \epsilon_{b} \in\left(0, \frac{1}{2}\right)$. Suppose that for all $\alpha \in\left[\alpha_{a}, \alpha_{b}\right]$, and for all $c, \tilde{c} \in \mathcal{T}_{N}$, one has

(0) $\chi_{a}(c) \chi_{b}(c)=0$,

(i) $\left\langle\chi_{a}(c)+\chi_{b}(c)\right\rangle_{N, \alpha} \geq A$,

(ii) $\left\langle\chi_{a}(c) \chi_{b}(\tilde{c})\right\rangle_{N, \alpha} \leq B$

and, meanwhile,

and

(iiia) $\left\langle\chi_{a}(c)\right\rangle_{N, \alpha_{a}}>1-\epsilon_{a}$

$\left(\right.$ iiib) $\left\langle\chi_{b}(c)\right\rangle_{N, \alpha_{b}}>1-\epsilon_{b}$.

Further, suppose that the above holds for all $N$ in some sequence $\mathcal{T}_{N} \nearrow \mathbb{Z}^{d}$. Then there is a value $\alpha_{t} \in\left(\alpha_{a}, \alpha_{b}\right)$ and two distinct (infinite volume) Gibbs states $\langle-\rangle_{\alpha_{t}}^{a}$ and $\langle-\rangle_{\alpha_{t}}^{b}$ (characterized, e.g. by the fact that $\left\langle\chi_{a}(c)\right\rangle_{\alpha_{t}}^{a} \geq 1-\delta$ and $\left\langle\chi_{b}(c)\right\rangle_{\alpha_{t}}^{b} \geq$ $1-\delta$, where $\delta$ is a particular function of $A$ and $B$ such that $\delta \rightarrow 0$ as $A \rightarrow 1$ and $B \rightarrow 0)$.

Proof. See, e.g. [KS] or [S]. We remark that the hypotheses (0)-(iii) as stated, in [S] pertain to actual infinite volume states. Here, since we are assuming that they hold for the states $\langle-\rangle_{N, \alpha}$ as $\mathcal{T}_{N} \nearrow \mathbb{Z}^{d}$, we may rest assured that the desired properties hold in the various limiting states. Inspecting the proof of Theorem 4 from $[\mathrm{KS}]$, one can ascertain that for $A=1-\eta$ and $B=\eta$, the function $\delta(A, B) \sim \sqrt{\frac{\eta}{2}}$.

\section{Proof of Theorem 2.1.}

In our analysis of the two dimensional Potts model there are few basic patterns which, in various regions of parameter space, will dominate the spin/particle configurations. We will define these following events by specifying the configurations restricted to an arbitrary $\Lambda \subset \mathcal{T}_{N}$ :

The empty event - all sites in $\Lambda$ are vacant,

$$
I_{\emptyset}(\Lambda)=\left\{n_{N}, \sigma_{N} \mid n_{i}=0 \text { for all } i \in \Lambda\right\}
$$

The disordered event - all sites in $\Lambda$ are occupied but all pairs of neighboring spins disagree,

$$
I_{d}(\Lambda)=\left\{n_{N}, \sigma_{N} \mid n_{i}=1 \text { for all } i \in \Lambda, \sigma_{i} \neq \sigma_{j} \text { for all } i, j \in \Lambda,|i-j|=1\right\} .
$$

The staggered events - the event " $A$ " with all the even sites of $\Lambda$ occupied and all the odd sites of $\Lambda$ vacant (cf. the equation 2.5 ),

$$
I_{A}(\Lambda)=\left\{n_{N}, \sigma_{N} \mid n_{i}=1 ; i \in \Lambda, i_{1}+i_{2} \text { even, } n_{i}=0 ; i \in \Lambda, i_{1}+i_{2} \text { odd }\right\} .
$$

and similarly for $B$ with the words even and odd exchanged.

And finally, the ordered event - all sites in $\Lambda$ occupied with all spins aligned,

$$
I_{o}(\Lambda)=\left\{n_{N}, \sigma_{N} \mid n_{i}=1 ; i \in \Lambda, \sigma_{i} \text { is constant throughout } \Lambda\right\} .
$$

As usual, we let $\chi_{\emptyset}(\Lambda), \chi_{d}(\Lambda), \chi_{A}(\Lambda), \chi_{B}(\Lambda)$, and $\chi_{o}(\Lambda)$ denote the indicators of the corresponding events.

In the forthcoming discussion, a given state will be characterized by the dom- 


\section{FiguRE 2.3}

of phase coexistence, the patterns of the relevant states are taken for generalized "ground states" and the remaining ones are considered to be part of contours. More precisely, let us choose a set $Q \subset\{\emptyset, d, o, A, B\}$ of labels for the states under consideration. For a given configuration $\left(n_{N}, \sigma_{N}\right)$, we say that the square $\tilde{c}$ is good if $\left(n_{\tilde{c}}, \sigma_{\tilde{c}}\right) \in I_{q}(\tilde{c})$ for some $q \in Q$. The remaining squares are called bad and any component, $\Gamma$, of their union is a contour of the configuration $\left(n_{N}, \sigma_{N}\right)$.

Of course even for a system as simple as the two-dimensional site-diluted Potts model, there are many possible modes of bad behavior. It turns out that more efficient estimates are obtained by taking finer characterizations of bad behavior and, in this case, we have taken things about as far as they can go. With the idea in mind to use the chessboard estimates of Lemma 2.3, let us define the restricted partition functions $\mathcal{Z}_{b}$ for a behavioral patterns $b$ via $\left\langle\chi_{b}\left(\mathcal{T}_{N}\right)\right\rangle_{N, \beta}^{J, \mu, \kappa} \equiv \frac{\mathcal{Z}_{b}}{\mathcal{Z}}$. (For simplicity, we usually omit explicit reference to the various parameters.) It is clear that we need bounds on several partition functions $\mathcal{Z}_{b}$. We urge that, rather than pouring over the formal definitions listed below, the reader immediately consults Figure 2.3.

Lemma 2.5. Consider, for two dimensions, the patterns $\emptyset, A, B, d$, and o as described previously. Then we have

$$
\begin{gathered}
\mathcal{Z}_{\emptyset}^{\frac{1}{\left|\mathcal{T}_{N}\right|}}=1, \\
\mathcal{Z}_{A}^{\frac{1}{\left|\mathcal{T}_{N}\right|}}=\mathcal{Z}_{B}^{\frac{1}{\left|\mathcal{T}_{N}\right|}}=e^{\frac{1}{2} \beta \mu} q^{\frac{1}{2}}, \\
e^{\beta \mu} e^{2 \beta \kappa} e^{-2 \beta J} \sqrt{q(q-4)} \leq \mathcal{Z}_{d}^{\frac{1}{\left|\mathcal{T}_{N}\right|}} \leq e^{\beta \mu} e^{2 \beta \kappa} e^{-2 \beta J} q, \\
\mathcal{Z}_{O}^{\frac{1}{\left|\mathcal{T}_{N}\right|}}=e^{\beta \mu} e^{2 \beta \kappa}\left(q^{\frac{1}{\left|\mathcal{T}_{N}\right|}}\right) .
\end{gathered}
$$

Furthermore, we have 
where $b_{1}$ is any of the four patterns on $c$ where a particular corner is the sole site occupied;

$$
\mathcal{Z}_{b_{2}^{(o)}}^{\frac{1}{\left|T_{N}\right|}} \lesssim e^{\frac{1}{2} \beta \mu} e^{\frac{1}{2} \beta \kappa}
$$

where $b_{2}^{(o)}$ is any of the four patterns on $c$ where a neighboring pair of sites is occupied and in alignment while the other two sites are vacant;

$$
\mathcal{Z}_{b_{2}^{(d)}}^{\frac{1}{\left|\mathcal{T}_{N}\right|}} \lesssim e^{\frac{1}{2} \beta \mu} e^{\frac{1}{2} \beta \kappa} e^{-\frac{1}{2} \beta J} q^{\frac{1}{2}}
$$

where $b_{2}^{(d)}$ is any of the four patterns on $c$ where a neighboring pair of sites is occupied by spin-states in disagreement and the other two sites are vacant;

$$
\mathcal{Z}_{b_{3}^{(o)}}^{\frac{1}{\left|\mathcal{T}_{N}\right|}} \lesssim e^{\frac{3}{4} \beta \mu} e^{\beta \kappa}
$$

where $b_{3}^{(o)}$ is any of the four patterns on $c$ where only one (particular) corner is vacant and the three occupied sites have their spins aligned;

$$
\mathcal{Z}_{b_{3}^{(d)}}^{\frac{1}{\left|\mathcal{T}_{N}\right|}} \lesssim e^{\frac{3}{4} \beta \mu} e^{\beta \kappa} e^{-\beta J} q^{\frac{3}{4}}
$$

where $b_{3}^{(d)}$ is any of the four patterns on c where only one (particular) corner is vacant and each spin disagrees with its neighbor;

$$
\mathcal{Z}_{b_{3}^{(m)}}^{\frac{1}{\mid T_{N}}} \lesssim e^{\frac{3}{4} \beta \mu} e^{\beta \kappa} e^{-\frac{1}{2} \beta J} q^{\frac{1}{4}}
$$

where $b_{3}^{(m)}$ is any of the eight patterns on $c$ where only one (particular) corner is vacant and the central site of the occupied trio is in alignment with one (particular) neighbor and is in disagreement with the other;

$$
\mathcal{Z}_{f_{1}}^{\frac{1}{\left|\mathcal{T}_{N}\right|}} \lesssim e^{\beta \mu} e^{2 \beta \kappa} e^{-\frac{3}{2} \beta J} q^{\frac{1}{2}}
$$

where $f_{1}$ is any one of the four patterns on $c$ where all sites are occupied, a particular neighboring pair is in alignment, and the other three neighboring pairs are in disagreement;

$$
\mathcal{Z}_{f_{2}^{\|}}^{\frac{1}{\left|\mathcal{T}_{N}\right|}} \lesssim e^{\beta \mu} e^{2 \beta \kappa} e^{-\beta J}
$$

where $f_{2}^{\|}$is either of the two patterns on $c$ where all sites are occupied and each site agrees with one of its neighbors and disagrees with the other;

$$
\mathcal{Z}_{f_{2}^{\perp}}^{\frac{1}{\left|\mathcal{T}_{N}\right|}} \lesssim e^{\beta \mu} e^{2 \beta \kappa} e^{-\beta J} q^{\frac{1}{4}}
$$

where $f_{2}^{\perp}$ is any one of the four patterns on $c$ where all sites are occupied and three 
In the above, $\lesssim$ indicates that the ratio of the left and right hand sides is bounded by unity in the limit $\mathcal{T}_{N} \nearrow \mathbb{Z}^{2}$ and we use $\left|\mathcal{T}_{N}\right| \equiv N^{2}$ as notation for the number of sites in $\mathcal{T}_{N}$.

Proof. The equalities for $\mathcal{Z}_{\emptyset}, \mathcal{Z}_{A}$ and $\mathcal{Z}_{B}, \mathcal{Z}_{o}$, as well as the upper bound on $\mathcal{Z}_{d}$ are obvious. The lower bound for $\mathcal{Z}_{d}$ follows from the observation that for any configuration on the even sublattice, each spin on the odd sublattice enjoys at least $(q-4)$ states that are guaranteed to be different from all of its neighbors.

We will not spell out of all the details for all of the other estimates; each case can be readily checked. For example, examining the site configuration that leads to $\mathcal{Z}_{b_{3}^{(m)}}$, it is seen that three quarters of the sites and one half of the neighboring pairs are present. This gives us a "prefactor" of $e^{\frac{3}{4} \beta \mu N^{2}} e^{\beta \kappa N^{2}}$. Half of these occupied neighboring pairs are in disagreement yielding the factor $e^{-\frac{1}{2} \beta J}$. What remains amounts to $\frac{N^{2}}{4}$ independent sites and $\frac{N}{2}$ independent (frozen) chains of length $N$ whose joint contribution is no more than $q^{\frac{1}{4} N^{4}+\frac{N}{2}}$. The bound on $\mathcal{Z}_{b_{3}^{(m)}}^{\frac{1}{\left|\mathcal{T}_{N}\right|}}$ follows immediately. The other estimates are obtained in similar fashion.

Proof of Theorem 2.1. First we observe that the Hamiltonian in the equation (2.2) is of the form described in Proposition 2.2 so we may use the chessboard estimates of the subsequent lemma. We will break our proof into separate proofs for the regions $R_{I}-R_{I V}$ and we will start with $R_{I I}$ since this case offers the fewest obstacles.

The region $R_{I I}$ is the cold temperature region and thus we anticipate that the dominant configurations on the square will be either $\emptyset$ or $o$. To estimate the probability of other configurations, we will therefore use only $\mathcal{Z}_{\emptyset}$ and $\mathcal{Z}_{O}$ as our denominator in the (single square) chessboard estimate. Evidently, we are anticipating that 1 and/or $e^{\beta \mu} e^{2 \beta \kappa}$ are large compared with "anything else" and we note that their relative size is determined by $\mu$ : the control parameter. Let us demonstrate explicitly in the case of $b_{3}^{(d)}$, the basic calculations for dispensing with the various patterns. Using the chessboard estimate, the relevant calculation from Lemma 2.3, and $\mathcal{Z} \geq \mathcal{Z}_{\emptyset}+\mathcal{Z}_{o}$, we arrive at

$$
\begin{gathered}
\left\langle\chi_{b_{3}^{(d)}}(c)\right\rangle_{N, \beta}^{J, \mu, \kappa} \lesssim \frac{e^{\frac{3}{4} \beta \mu} e^{\beta \kappa} e^{-\beta J} q^{\frac{3}{4}}}{\left[1+\left(e^{\beta \mu} e^{2 \beta \kappa}\right)^{N^{2}}\right]^{\frac{1}{N^{2}}}}= \\
=e^{-\beta J} q^{\frac{3}{4}} e^{-\frac{1}{2} \beta \kappa}\left[\frac{e^{\frac{3}{4} \beta \mu} e^{\frac{3}{2} \beta \kappa}}{\left[1+\left(e^{\beta \mu} e^{2 \beta \kappa}\right)^{N^{2}}\right]^{\frac{1}{N^{2}}}}\right] .
\end{gathered}
$$

Observe that the term in the square brackets is bounded above by unity independent of the value of $e^{\beta \mu} e^{2 \beta \kappa}$. Thus we arrive at $\left\langle\chi_{b_{3}^{(d)}}(c)\right\rangle_{N, \beta}^{J, \mu, \kappa} \lesssim q^{\frac{3}{4}} e^{-\beta J} e^{-\frac{1}{2} \beta \kappa}$.

Let us recall that $J>\kappa$ and define the region $R_{I I}$ by the condition

$$
R_{I I}: \quad q^{\frac{1}{4}} e^{-\frac{1}{2} \beta \kappa} \leq a_{I I}
$$

where $a_{I I}$ is a small number that we will specify shortly. Evidently, throughout $R_{I I}$, we have $\left\langle\chi_{b_{3}^{(d)}}(c)\right\rangle_{N, \beta}^{J, \mu, \kappa} \lesssim a_{I I}^{3}$. Similar estimates can be performed for all the quantities with the results that the probabilities for observing examples of the patterns $b_{1}, b_{2}^{(o)}, b_{3}^{(o)}$ and $f_{2}^{\perp}$ on the unit square are all (asymptotically) bounded above by $a_{I I}$, the probabilities for observing examples of the patterns $A, B, b_{2}^{(d)}$ or 
Thus, for $a_{I I}$ sufficiently small — which here simply means $\beta$ large — we have verified (i) of Lemma 2.4. (We note that the multiplicity of each pattern must be taken into account in order to perform an honest calculation of $a_{I I}$.) Clearly $(0)$ is satisfied and, along any line of constant $\beta$ in $R_{I I}$, (iiia) and (iiib) are satisfied for $\pm \mu$ sufficiently large. The remaining issue is therefore (ii), and here we get into the contour estimates.

Suppose that $I_{o}(c)$ and $I_{\emptyset}(\tilde{c})$ both occur with $\tilde{c} \neq c$. Let us consider, e.g. the connected component of "ordered sites" that contains $c$. The boundary of this region may be defined, on the dual lattice, by drawing a bond between all pairs of neighboring sites that have one member in the region and the other member out. It is clear that these bonds form closed loops and, furthermore, a moments thought reveals that the endpoints of these bonds always reside in the middle of bad squares. Thus (for $a_{I I}$ small) we can use the chessboard estimate to show that the probability of a contour goes to zero exponentially fast in its length. When we have both $I_{o}(c)$ and $I_{\emptyset}(\tilde{c})$ in the same configuration, there must be either a contour "surrounding" one or the other region or there must be a long contour (i.e. of length exceeding $N$ ) which wraps around the torus and separates the two squares. In any case, we may now use the standard (Ising type) Peierls estimate to establish condition (ii) in Lemma 2.4.

Thus, for any $\beta$ satisfying the condition in the equation $(2.14)-$ with $a_{I I}$ sufficiently small - the conclusions of Lemma 2.4 hold with $\mu$ serving as the parameter $\alpha$. For such a fixed $\beta$, let $\mu_{t}$ be any value of the parameter from Lemma 2.4 at which phase coexistence occurs and let $\langle-\rangle_{\beta, \mu_{t}}^{d}$ and $\langle-\rangle_{\beta, \mu_{t}}^{o}$ denote the associated coexisting states. Below we show that, in fact, the point $\mu_{t}$ is uniquely determined. Observe that in the states $\langle-\rangle_{\beta, \mu_{t}}^{d}$ and $\langle-\rangle_{\beta, \mu_{t}}^{o}$ we have

$$
\left\langle\chi_{\emptyset}(c)\right\rangle_{\beta, \mu_{t}}^{d}>1-\delta
$$

and

$$
\left\langle\chi_{o}(c)\right\rangle_{\beta, \mu_{t}}^{o}>1-\delta,
$$

for all $c$ and with a "small" $\delta=\delta\left(a_{I I}, q\right)$. It therefore follows that, for any $i$, one has

$$
\left\langle n_{i}\right\rangle_{\beta, \mu_{t}}^{d}<\delta
$$

and

$$
\left\langle n_{i}\right\rangle_{\beta, \mu_{t}}^{o}>1-\delta
$$

Since the density is a thermodynamic observable (i.e., it may be obtained via a derivative of the free energy), it follows from the standard convexity arguments that, in any limiting Gibbs state corresponding to $(\beta, \mu)$ with $\mu>\mu_{t}$, the density exceeds $1-\delta$ (and hence $\chi_{d}$ has a small average value). Similarly, in any Gibbs state corresponding to $\mu<\mu_{t}$, the density is less than $\delta$ (and $\chi_{o}$ has small average). Thus, we may unambiguously define the old $\mu_{t}$ as $\mu_{I I}(\beta)$, the unique point of coexistence of high and low density states along the associated isotherm. Construction of the $q$ individual magnetized states states for $\mu \geq \mu_{I I}(\beta)$ follows from standard procedure, e.g. as in Theorem 2 of [KS].

We now show that $\mu_{I I}(\beta)$ enjoys certain monotonicity property, namely, that $\beta \mu_{I I}(\beta)$ is monotone in $\beta$. Let $\beta_{1}>\beta_{2}$ and let $\mu<\mu_{I I}(\beta)$; we will show that 
$\langle-\rangle_{N, \beta^{\prime}}^{J, \mu^{\prime}, \kappa}$. Using the same convexity-type arguments, it is clear that if we decrease $\beta$ while keeping $\beta \mu$ fixed, the average of $n_{i} n_{j}\left(\delta_{\sigma_{i}, \sigma_{j}}-1\right)+\frac{\kappa}{J} n_{i} n_{j}$ will only decrease. Explicitly, for all neighboring pairs $\langle i, j\rangle$, we have

$$
\left\langle n_{i} n_{j}\left(\delta_{\sigma_{i}, \sigma_{j}}-1\right)+\frac{\kappa}{J} n_{i} n_{j}\right\rangle_{\beta_{2}, \frac{\beta_{1}}{\beta_{2}} \mu}^{*} \leq\left\langle n_{i} n_{j}\left(\delta_{\sigma_{i}, \sigma_{j}}-1\right)+\frac{\kappa}{J} n_{i} n_{j}\right\rangle_{\beta_{1}, \mu}^{*} .
$$

Neglecting the non positive term on the right hand side and using $n_{i} \geq n_{i} n_{j}$, we may replace the upper bound with $\delta \frac{J}{\kappa}$ since $\mu<\mu_{I I}\left(\beta_{1}\right)$. Thus the left hand side is "small" and we claim (for $\delta$ sufficiently small) that this implies that the density $\left\langle n_{i}\right\rangle_{\beta_{2}, \frac{\beta_{1}}{\beta_{2}} \mu}^{*}$ is also small. The claim follows from the fact that for any $(\beta, \mu) \in R_{I I}$, the quantities $\left\langle n_{i} n_{j}\left(\delta_{\sigma_{i}, \sigma_{j}}-1\right)\right\rangle_{\beta, \mu}^{*}$ and $\left\langle n_{i}\left(1-n_{j}\right)\right\rangle_{\beta, \mu}^{*}$ are uniformly small - indeed, the fact that $n_{i} n_{j}\left(\delta_{\sigma_{i}, \sigma_{j}}-1\right)=1$ or $n_{i}\left(1-n_{j}\right)=1$ forces the indicator of some bad square to be one. Hence, we may write

$$
\left\langle n_{i}\right\rangle_{\beta_{1}, \mu}^{*}<c \delta
$$

with $c$ a constant of order of unity. From this it follows (for sufficiently small $\delta$ one has $c \delta<1-\delta)$ that

$$
\beta_{1} \mu \leq \beta_{2} \mu_{I I}\left(\beta_{2}\right)
$$

which is the desired statement.

Finally, we will show that $\mu_{I I}(\beta)$ is continuous, and hence that there is a continuous phase boundary. Suppose that $\mu>\mu_{I I}(\beta)$. It follows that for any $\beta^{\prime}>\beta$, in any Gibbs state $\langle-\rangle_{\beta^{\prime}, \mu}^{*}$ the particle density exceeds $1-\delta$. However, this means (see, e.g. [G], Theorem 4.23) that we may construct a limiting Gibbs state $\langle-\rangle_{\beta, \mu}^{*}$, for which the density is also at least as large as $1-\delta$. This in turn implies that $\mu \geq \mu_{I I}(\beta)$ and hence that $\mu_{I I}(\beta)$ is lower semicontinuous. Upper semicontinuity of $\mu_{I I}(\beta)$ follows from an essentially identical argument.

Next, we turn attention to $R_{I}$ where the dominant patterns are expected to be $d$ and $o$. The control parameter is, as expected, $q e^{-2 \beta J}$. (I.e. if this is small, $o$ dominates and if it is large, $d$ dominates.) It turns out that the small quantity that defines $R_{I}$ is $q^{\frac{1}{4}} e^{-\frac{1}{4} \beta \mu} e^{-\frac{1}{2} \beta \kappa}$; we define

$$
R_{I}: \quad q^{\frac{1}{4}} e^{-\frac{1}{4} \beta \mu} e^{-\frac{1}{2} \beta \kappa} \leq a_{I}
$$

with $a_{I}$ sufficiently small as later requirements will dictate. Unlike the previous case, here we will also require $q$ itself to be large (actually $q^{\frac{1}{4}}$, c.f. below) in particular so that the control parameter can swing from large to small. In $R_{I}$, the principal culprits are the $b_{3}^{(d)}$ pattern, the probability of whose appearance on $c$ is bounded by $a_{I}$, and $f_{1}$ and $f_{2}^{\perp}$, each of which gets a factor of $q^{-\frac{1}{4}}$. All the other patterns lead to bounds involving higher powers of $a_{I}$ and/or $q^{-\frac{1}{4}}$. For possible future reference, we will tabulate the results. We obtain $\emptyset: a_{I}^{4} ; A$ and $B: a_{I}^{2} ; b_{1}: a_{I}^{3} q^{-\frac{1}{2}}$; $b_{2}^{(o)}: a_{I}^{2} q^{-\frac{1}{2}} ; b_{2}^{(d)}: a_{I}^{2} q^{-\frac{1}{4}} ; b_{3}^{(o)}$ and $b_{3}^{(m)}: a_{I} q^{-\frac{1}{4}}$ and finally $f_{2}^{\|}: q^{-\frac{1}{2}}$. To ensure that all of $R_{I}$ is covered, we will perform our variant of Proposition 2.4 along curves where $\beta \mu+2 \beta \kappa$ is constant; we will denote this quantity $\omega$. Evidently, along each curve of constant $\omega$ (sufficiently large), we can find a $\beta_{t}$ and a pair of coexisting states, $\langle-\rangle_{\beta_{t}, \mu}^{d}$ and $\langle-\rangle_{\beta_{t}, \mu}^{o}$ with, e.g., $\left\langle\chi_{o}\right\rangle_{\beta_{t}, \mu}^{d} \leq \delta$ and $\left\langle\chi_{o}\right\rangle_{\beta_{t}, \mu}^{o} \geq 1-\delta$. The 
the "thermodynamic" variables are somewhat artificial. Writing the Hamiltonian in terms of the parameters $\omega$ and $\beta$, it is evident that, along curves of constant $\omega$, the quantity $X$ corresponding to $\frac{1}{N^{2}} \sum_{\langle i, j\rangle}\left[n_{i} n_{j}\left(\delta \sigma_{i}, \sigma_{j}-1\right)+\frac{\kappa}{j} n_{i}\left(1-n_{j}\right)\right]$ is conjugate to the parameter $\beta$. We will now argue that this quantity takes a jump at $\beta=\beta_{t}$. Indeed, in both states, the average of $n_{i}\left(1-n_{j}\right)$ is small and thus, as is fairly easily seen,

$$
\langle X\rangle_{\beta_{t}, \mu}^{o} \geq-c_{1} \delta
$$

while

$$
\langle X\rangle_{\beta_{t}, \mu}^{d} \leq-\left(1-c_{2} \delta\right)
$$

with $c_{1}$ and $c_{2}$ (positive) constants of order unity. Thus, in all Gibbs states with the same $\omega$ and $\beta>\beta^{\prime}\left(\beta<\beta^{\prime}\right)$, the mean values of $X$ are not smaller than $-c_{1} \delta$ (not greater than $-\left(1-c_{2} \delta\right)$ ). Since, throughout $R_{I}$, the mean of $n_{i}\left(1-n_{j}\right)$ is uniformly small, for those states that emerge as limits of $\langle-\rangle_{N, \beta}^{\kappa, \mu, J}$, only one large jump of the mean of $X$ is possible. This defines, unambiguously, $\beta_{I}(\omega)$. By the above reasoning, it is easy to see that whenever $\beta<\beta_{I}(\omega)$, no state with parameters $\beta, \omega$ can have an appreciable average value of $\chi_{o}$ and for $\beta>\beta_{I}(\omega)$ no state have an appreciable value of $\chi_{d}$.

Let us now formally express our phase plane coordinates in terms of $\omega$ and $\eta=\beta \mu$, and write our function as $\eta_{I}(\omega)$. (Explicitly: $\eta_{I}(\omega)=\omega-2 \beta_{I}(\omega) \kappa$.) Following identically the (more physically appealing) arguments of $R_{I I}$, it is clear that $\eta_{I}(\omega)$ is monotone increasing and, finally, continuous.

Let us now dispense with the region $R_{I I I}$. Our definition of the region is given by

$$
R_{I I I}: \quad q^{\frac{1}{2}} e^{-\beta J} \leq g_{I I I} \text { and } q^{-\frac{1}{4}} e^{-\frac{1}{4} \beta \mu} \leq a_{I I I}
$$

with $a_{I I I}$ and $g_{I I I}$ sufficiently small numbers.

The dominant patterns are anticipated to be $o$ or $A / B$ and hence the control parameter is $\mathcal{P} \equiv q^{-\frac{1}{2}} e^{\frac{1}{2} \beta \mu} e^{2 \beta \kappa}$. In $R_{I I I}$, we obtain the following bounds on the probability of observing the stated patterns: $\emptyset: a_{I I I}^{2} ; d: g_{I I I}^{2} ; \quad b_{1}: a_{I I I}$; $b_{2}^{(o)}: q^{-\frac{1}{4}} a_{I I I}^{\frac{1}{2}} ; \quad b_{2}^{(d)}: a_{I I I}^{\frac{1}{2}} g_{I I I}^{\frac{1}{2}} ; \quad b_{3}^{(o)}: q^{-\frac{1}{4}} ; \quad b_{3}^{(d)}: g_{I I I} ; \quad b_{3}^{(m)}: g_{I I I} q^{-\frac{1}{2}} ;$ $f_{1}: g_{I I I}^{\frac{3}{2}} q^{-\frac{1}{4}} ; f_{2}^{\|}: g_{I I I}^{\frac{1}{2}} q^{-\frac{1}{4}}$, and $f_{2}^{\perp}: g_{I I I}^{\frac{1}{2}} q^{-\frac{1}{4}}$.

Clearly, $\mathcal{P}$ can take on large values in $R_{I I I}$ since since it is infinite on the $\beta \mu=\infty$ boundary of the region. This (re)proves the existence of ordered phases in $R_{I I I}$. To establish the existence of staggered phases, it must be shown that $\mathcal{P}$ can take on small values in $R_{I I I}$. Indeed, placing ourselves in the farthest corner of $R_{I I I}$ : $q^{\frac{1}{2}} e^{\beta_{0} J}=g_{I I I}$ and $q^{-\frac{1}{4}} e^{-\frac{1}{4} \beta_{0} \mu_{0}}=a_{I I I}$, we find

$$
\mathcal{P}\left(\beta_{0}, \mu_{0}\right)=\left(\frac{1}{a_{I I I}}\right)^{2}\left(\frac{1}{g_{I I I}}\right)^{\frac{2 \kappa}{J}} q^{-\left(1-\frac{\kappa}{J}\right)} .
$$

Using, in this last step, the condition that $\kappa<J$, we see that for very large $q$, there is a staggered phase in $R_{I I I}$. Actually, for the purposes of our subsequent discussion, it is assumed that $q$ is large enough to ensure that staggered phases exist in a neighborhood of $\left(\beta_{0}, \mu_{0}\right)$.

Let us begin our analysis of the phase boundary by discussing the behavior along the curve $q^{-\frac{1}{4}} e^{-\frac{1}{4} \beta \mu}=a_{I I I}$. By applying Proposition 2.4, there is a point $\beta_{t}, \mu_{t}$ on this curve at which there coexist Gibbs states $\langle-\rangle_{\beta_{t}, \mu_{t}}^{o}$ and $\langle-\rangle_{\beta_{t}, \mu_{t}}^{S}, S=A, B$ 
We now claim that $\mu_{t}, \beta_{t}$ divides this curve into a staggered portion and ferromagnetic portion: in particular, if $(\beta, \mu) \in R_{I I I}, \beta \mu=\beta_{t} \mu_{t}$, and $\beta>\beta_{t}$, and $\langle-\rangle_{\beta, \mu}^{*}$ is any Gibbs state that emerges from the torus states, $\left\langle\chi_{o}\right\rangle_{\beta, \mu}^{*}>1-c \delta$ with a similar statement holding for $\beta<\beta_{t}$. On the basis of proviso following the equation (2.26), it is clear that $\beta_{t}>\beta_{0}$.

Let us now investigate the behavior along isotherms that lie in $R_{I I I}$. To facilitate our analysis, we may again consider the particle density. It is clear that if $\beta \geq \beta_{t}$, then for all $\mu$ (with $(\beta, \mu) \in R_{I I I}$ ), the particle density in any Gibbs state is close to unity. This rules out the possibility of a state with an appreciable average value of either of the staggered order parameters and, further demonstrates the existence of states in which the average of $\chi_{o}(c)$ is close to unity. On the other hand, if $\beta<\beta_{t}$, we first reemphasize that at $\mu=\mu_{0} \frac{\beta_{0}}{\beta}$ (i.e. along the bottom boundary of $R_{I I I}$ ) the average of $\left\langle\chi_{A}(c)+\chi_{B}(c)\right\rangle_{\beta, \mu}^{*}$ is close to one in any state $\langle-\rangle_{\beta, \mu}^{*}$ which is a limit of torus states $\langle-\rangle_{N, \beta}^{\kappa, \mu, J}$. Thus, using Proposition 2.4, we can find a $\mu_{t}$ such that at $\left(\beta, \mu_{t}\right)$, staggered states and ordered states (with order parameters close to unity) coexist.

For $\mu>\mu_{t}$, the reasoning is identical to the analysis of the isotherms with $\beta \geq \beta_{t}$. Furthermore, it is not difficult to see that if $\mu<\mu_{t}$, there cannot exist any Gibbs states in which the average of $\chi_{0}$ is close to unity. This permits us to unambiguously define a function $\mu_{I I I}(\beta)\left(\beta_{0} \leq \beta<\beta_{t}\right)$. Nevertheless, it still remains to be shown that for $(\mu, \beta)$ with $\mu<\mu_{I I I}(\beta)$ there exists staggered states with order parameter close to unity. To this end, observe that in the staggered states at $\left(\beta, \mu_{I I I}(\beta)\right)$, the particle density is not appreciably larger than $\frac{1}{2}$. It follows that in any limiting state of $\langle-\rangle_{N, \beta}^{J, \mu, \kappa}$ with $\mu<\mu_{I I I}(\beta)$, the average of $\chi_{o}(c)$ is also not much larger than $\frac{1}{2}$ : indeed, if $\langle i, j\rangle$ is in $c$,

$$
\chi_{o}(c) \leq n_{i} n_{j} \leq \frac{1}{2}\left(n_{i}+n_{j}\right)
$$

and the average of $\frac{1}{2}\left(n_{i}+n_{j}\right)$ (in any limiting torus state) cannot exceed the density in, e.g. the state $\langle-\rangle_{\beta, \mu_{I I I}(\beta)}^{S}$. Since, in $R_{I I I}$, the sum $\chi_{o}+\chi_{A}+\chi_{B}$ has average close to unity and $\left\langle\chi_{A}(c)\right\rangle_{N, \beta}^{J, \mu, \kappa}=\left\langle\chi_{B}(c)\right\rangle_{N, \beta}^{J, \mu, \kappa}$, it follows that

$$
\liminf _{N \rightarrow \infty}\left\langle\chi_{A}(c)\right\rangle_{N, \beta}^{J, \mu, \kappa} \geq g
$$

with $g \approx \frac{1}{4}$ for every square $c$ on the torus. Next, for any sequence $\tilde{c}_{N}$ located at distance at least $N / 2$ from the origin, we will consider the conditional measures $\left\langle-\mid \chi_{A}\left(\tilde{c}_{N}\right)=1\right\rangle_{N, \beta}^{\kappa, \mu}$. If $c$ is a fixed square, we may write

$$
\left\langle\chi_{A}(c) \mid \chi_{A}\left(\tilde{c}_{N}\right)=1\right\rangle_{N, \beta}^{J, \mu, \kappa}=1-\left\langle\sum_{b \neq A} \chi_{b}(c) \mid \chi_{A}\left(\tilde{c}_{N}\right)=1\right\rangle_{N, \beta}^{J, \mu, \kappa} \geq 1-\frac{1}{g} \delta
$$

uniformly in $N$, for $N$ sufficiently large. Restricting the measures on the left hand side to any sequence $V_{N}$ centered at origin and of the diameter less than $\frac{N}{2}$, the existence of the desired staggered states is established.

Finally, mimicking the argument used along the line $\beta \mu=\beta_{0} \mu_{0}$, it is immediately clear that $\mu_{I I I}(\beta)$ is monotone decreasing in $\beta$; continuity of this function is 
Let us now derive conditions that ensure complete analyticity. We will consider a one site system with a boundary condition $B$ as provided by the state of its four neighbors. We will denote this state by $\rho_{B}(-)$ and abbreviate its argument by $0,1, \ldots q$. If $B^{\prime}$ is a boundary condition that differs from $B$ at just one boundary site, it is sufficient, by [DS], to demonstrate that

$$
\max _{B} \sum_{k} \max _{B^{\prime}}\left|\rho_{B}(k)-\rho_{B^{\prime}}(k)\right| \leq \frac{1}{4} .
$$

It is straightforward to show that the above is always small provided that $q e^{\beta \mu} e^{4 \beta \kappa}$ or $q\left|1-e^{ \pm \beta J}\right|$ is small. We will illustrate this in the case where an occupied boundary spin changes from the state 1 to the state 2 . Let us define the normalization, in the obvious fashion, so that the weight of the empty state is always one. Comparing the weights, $\left(w_{B}(0), w_{B}(1), \ldots w_{B}(q)\right)$, before and $\left(w_{B^{\prime}}(0), w_{B^{\prime}}(1), \ldots w_{B^{\prime}}(q)\right)$, after the change, we see that $w_{B}(0)=w_{B}(0), w_{B^{\prime}}(1)=w_{B}(1) e^{-\beta J}, w_{B^{\prime}}(2)=w_{B}(2) e^{+\beta J}$ and $w_{B^{\prime}}(k)=w_{B}(k)$ for all $k>2$. Thus, there is little effect if either $\beta J$ is small or the $w(k)$ 's themselves are small for $k>0$. Since, for $k>0$, the $w(k)$ 's are bounded above by $e^{\beta \mu} e^{4 \beta \kappa}$, this is easily implied in the region

$$
R_{I V}: q e^{\beta \mu} e^{4 \beta \kappa} \leq a_{I V} \text { or } q\left(1-e^{-\beta J}\right) \leq g_{I V}
$$

with $g_{I V}$ and $a_{I V}$ sufficiently small numbers. The other required changes in other states are similarly seen to be sufficiently small in the region $R_{I V}$.

All the features in the statement of this theorem (but not all the features of interest) have now been covered and we are finally finished.

Remark. The endpoints of the order-disorder boundaries are well understood. In particular, at $\beta=\infty$, there is (trivially) a transition at $\mu=-2 \kappa$. With some additional effort $[\mathrm{M}, \mathrm{Z}]$ at $\mu=\infty$, it can be shown that for large $q$, there is a $\beta_{t}(q)$ satisfying $q e^{-2 \beta_{t} J}=1+O\left(\frac{1}{q}\right)$ which is the unique point for order/disorder coexistence (and no other phases present). On the basis of these facts, we can prove:

Corollary. In both the temperature and chemical potential variables, the staggered phases are reentrant.

Proof. For the case of the temperature parameter, all we have to do is show that the "small $\mathcal{P}$ " regime of $R_{I I I}$ intersects the line $\mu=-2 \kappa$. Let us place ourselves at the right boundary of $R_{I I I}: q^{\frac{1}{2}} e^{-\beta J}=g_{I I I}$ and show that the intersection of this boundary with the above mentioned line takes place in $R_{I I I}$. To do this, all that we need do is demonstrate that here, $q^{\frac{1}{4}} e^{\frac{1}{4} \beta \mu}$ is large; having done this, we will then have automatically demonstrated $\mathcal{P}$ is small (since $\mu=-2 \kappa$ ). Now at this point in the phase plane, it is easily seen that $q^{\frac{1}{4}} e^{\frac{1}{4} \beta \mu}=g_{I I I}^{\frac{1}{2} \frac{\kappa}{J}} q^{\frac{1}{4}\left(1-\frac{\kappa}{J}\right)}$. In light of the equation (2.26), this is indeed small.

For the case of the fugacity parameter, the definition of $R_{I I I}$ precludes the above sort of result. However in our estimates we had to allow for the possibility that $\mathcal{P}$ was large or small. All of the patterns that require $q e^{-2 \beta J}$ small - the disordered patterns - have additional (hidden) factors of $\mathcal{P}$ which we can now bring into play. Let us therefore make the alternative assumptions that $q \frac{1}{2} e^{-\beta J} \leq g$ with $g \gtrsim 1$ and 
only three calculations that need to be redone, the results of which are $b_{3}^{(d)}: g \mathcal{P}^{\frac{1}{2}}$; $b_{2}^{(d)}: a_{I I I}^{\frac{1}{2}} g \mathcal{P}^{\frac{1}{4}}$; and, obviously, the disordered pattern $d$ which enjoys the bound of $g^{2} \mathcal{P}$. Now, all we need to do is show that the region where $\mathcal{P}$ is small (or, to be more precise, $\mathcal{P}^{\frac{1}{2}}$ is small) and the region $q^{-\frac{1}{4}} e^{-\frac{1}{4} \beta \mu} \leq a_{I I I}$ intersects the line $q \frac{1}{2} e^{-\beta J}=g \approx 1$. However, if we go to the "corner" we find that the calculation for $\mathcal{P}$ is the same as the one leading to the equation (2.26) with $g_{I I I}$ replaced by g. This is indeed small enough. 


\section{Staggered Phases in AN EXtended ClASs of Site Diluted Models}

In this section we will prove the existence of staggered phases for a general class of nearest neighbor site diluted models on $\mathbb{Z}^{d}$. Our results will be sufficiently general to preclude the global results for the whole of the phase diagram that were featured in the preceding section. Indeed, here we will treat systems in which a low temperature phase may be absent altogether.

Here we assume that the formal Hamiltonian for the uniform system takes the form

$$
H_{u}=-\sum_{\langle i, j\rangle} J\left(\sigma_{i}, \sigma_{j}\right)
$$

with $\sigma_{i} \in\{1, \ldots, q\}$ and $J\left(\sigma_{i}, \sigma_{j}\right)=J\left(\sigma_{j}, \sigma_{i}\right) \geq 0$.

The annealed site-diluted Hamiltonian on the torus $\mathcal{T}_{N}$ takes the form

$$
H_{N}\left(n_{N}, \sigma_{N}\right)=-\sum_{\langle i, j\rangle} n_{i} n_{j} J\left(\sigma_{i}, \sigma_{j}\right)-\mu \sum_{i} n_{i}-\kappa \sum_{\langle i, j\rangle} n_{i} n_{j}
$$

where $\mu, \kappa$, and the $n_{i}(\in\{0,1\})$ play the same roles as their counterparts in Section 2. In these models, we will not require that the a priori weights, $(w(1), \ldots, w(q))$, that determine the single spin distribution, are all equal. (However, to maintain continuity with what has preceded, we shall assume that the weights sum to $q$.)

Thus, the partition function on $\mathcal{T}_{N}$ is given by

$$
\mathcal{Z}_{N, \beta}=\sum_{\substack{n_{i}=0,1 \\ i \in \mathcal{T}_{N}, 1}} \sum_{\substack{\sigma_{i} \in\{1, \ldots, q\} \\ i \in \mathcal{T}_{N} ; n_{i}=1}} \exp \left\{-\beta H_{N}\left(n_{N}, \sigma_{N}\right)\right\} \prod_{i \in \mathcal{T}_{N} ; n_{i}=1} w_{i}\left(\sigma_{i}\right)
$$

As was the case in the two-dimensional Potts models, the problems of merit typically have $\kappa \geq 0$. Nevertheless, we can incorporate the cases $\kappa<0$ with little enough effort. The principal "small parameter" in the problem is the quantity

$$
s(\beta)=\max _{\left\{\sigma_{i}\right\}} w_{i}\left(\sigma_{i}\right) \sum_{\sigma_{j}} w_{j}\left(\sigma_{j}\right) e^{-\beta J\left(\sigma_{i}, \sigma_{j}\right)} .
$$

To prove staggered order for $\kappa>0$ (but not terribly large) our basic requirements are that $q$ is large, and that $s$ is small relative to $q$. A glance at the equation (3.4) shows that the condition $s \ll q$ is the statement that for an interacting pair of spins, not all the spin space is taken up by the lowest energy states. For $\kappa<0$, there is always staggered order at sufficiently low temperature (cf. example 2 below).

Our result can now be formulated precisely:

Theorem 3.1. Let $H_{N}$ denote the Hamiltonian in the equation (3.2) and suppose there is an inverse temperature $\beta^{*}$ and an $\epsilon \leq 1$ such that at $\beta=\beta^{*}$ the following two conditions hold:

(i) while

(ii)

$$
s\left(\beta^{*}\right) \leq q^{\epsilon},
$$

$$
q^{(1-\epsilon)} e^{-D \beta^{*} \kappa}>P
$$

with $P$ a fixed sufficiently large number and

$$
D=\left\{\begin{array}{l}
1 \text { if } \kappa<0, \\
\text { in }
\end{array}\right.
$$


Then there is a region $R$, which includes a neighborhood of the point $(\beta, \mu)=$ $\left(\beta^{*},-\left[D \kappa+\frac{\epsilon \log q}{\beta^{*}}\right]\right)$, such that for all $(\beta, \mu)$ in $R$, the set of infinite volume extremal Gibbs states corresponding to the Hamiltonian $H_{N}$ with these parameters contains (at least) two extremal elements $\langle-\rangle_{\beta, \mu}^{A}$ and $\langle-\rangle_{\beta, \mu}^{B}$. These states are characterized by distinctive staggered orderings: in particular, in the $A / B$ state, the even/odd sublattice has an occupation density in excess of the odd/even sublattice.

Examples.

(1) Generalized q-state ferromagnet.

Let $E_{q} \subset \mathbb{R}^{\nu}$ for some $\nu \leq q$ be a discrete set containing $q$ points which, without loss of generality, satisfies $|\boldsymbol{\sigma}|<1$ for all $\boldsymbol{\sigma} \in E_{q}$. The a generalized $q$-state ferromagnet may be constructed by writing $J\left(\boldsymbol{\sigma}_{i}, \boldsymbol{\sigma}_{j}\right)=\mathcal{J}\left(\boldsymbol{\sigma}_{i} \cdot \boldsymbol{\sigma}_{j}-1\right)$ with $\mathcal{J} \geq 0$. Further, letting $\boldsymbol{h} \in \mathbb{R}^{\nu}$ to denote the "external field", we may write

$$
w(\boldsymbol{\sigma}) \propto e^{\boldsymbol{h} \cdot \boldsymbol{\sigma}} .
$$

Then, if $q$ is sufficiently large, it is possible to find a $\kappa_{0}>0$ and $h_{0}>0$ such that for all $\kappa$ and $\boldsymbol{h}$ with $\kappa<\kappa_{0}$ and $\|\boldsymbol{h}\|<h_{0}$, the conditions of Theorem 3.2 are satisfied.

(2) Any q-state spin system with $\kappa<0$.

Consider any Hamiltonian of the form of the equation (3.2) with $\kappa<0$. The condition is satisfied for $\epsilon=1$ and $\beta$ sufficiently large.

Remark. Observe that for the Potts models, $s=\left(1+(q-1) e^{-\beta J}\right)$. Thus (i) and (ii) cannot both be satisfied unless $J \geq d \kappa$. Needless to say, in the two-dimensional analysis all that was required was $J>\kappa$ (and $q$ correspondingly large). Thus, clearly, we are working with a condition that is stronger than optimal. To obtain better conditions on $s$ (e.g. replacing $s$ by $s^{d}$ in (ii)) would require the detailed classification of all modes of "bad behavior" on a hypercube as in Lemma 2.5. This is an arduous task for the general case in $d \geq 3$. However, in any particular case, the details can be worked out with enough effort.

To prove Theorem 3.1, we again examine all possible behaviors on a (hyper)cube c. Our principal goal will be to establish that, in a certain range of parameters, the (staggered) patterns $A$ and $B$ are dominant.

The following estimates will form the core of our analysis.

Lemma 3.2. Let $n_{c}$ denote the occupation pattern restricted to the cube $c$ and $\chi_{n_{c}}\left(n_{N}, \sigma_{N}\right)$ be the indicator for the event that $n_{c}$ is the occupation pattern on $c$. Let $\mathrm{E}$ denote the number of bonds ("edges") in the configuration $n_{c}$ :

$$
\mathrm{E}=\#\left\{\text { pairs }\langle i, j\rangle \in c \mid n_{i}=n_{j}\right\},
$$

let $\mathrm{L}$ denote the number of (locked) sites that participate in the formation of bonds

$$
\mathrm{L}=\#\left\{\text { sites } i \in c \mid n_{i}=1 \text {, there exists } j \in c,|i-j|=1, n_{j}=1\right\},
$$

and $\mathrm{U}$ the number of unencumbered sites

$$
\mathrm{U}=\#\left\{\text { sites } i \in c \mid n_{i}=1, n_{j}=0 \text { for all } j \in c \text { with }|i-j|=1\right\} \text {. }
$$

Then

$$
\left\langle\chi_{n_{c}}\right\rangle_{N \beta}^{\kappa, \mu} \lesssim\left(\frac{1}{\tau^{\prime}}\right)^{\frac{1}{N^{d}}}\left[q^{\mathrm{u}} e^{\mathrm{U} \beta \mu} e^{\mathrm{L} \beta \mu} e^{2 \mathrm{E} \beta \kappa} s^{\mathrm{L}}\right]^{\frac{1}{2^{d}}}
$$


where $\langle-\rangle_{N, \beta}^{\kappa, \mu}$ denotes the torus Gibbs state for the Hamiltonian in the equation (3.2) and the symbol $\lesssim$ has the same meaning as in the statement of Lemma 2.5.

Proof. It is noted that the $H_{N}$ are of the form described in Proposition 2.2 so we may freely use the arsenal of RP techniques. According to the chessboard estimates of Lemma 2.3, it is clear that we must estimate the constrained partition functions $\mathcal{Z}_{n_{c}} \equiv \mathcal{Z}_{N, \beta}\left\langle\chi_{n_{c}}\right\rangle_{N, \beta}^{\kappa, \mu}$.

First observe that each free site in the periodic continuation of $n_{c}$ gives rise to a factor of $q e^{\beta \mu}$ and there are exactly $\left(\mathrm{U} / 2^{d}\right) N^{d}$ such sites. This accounts for the factor of $\left(q^{\mathrm{u}} e^{\mathrm{u} \beta \mu}\right)^{\frac{1}{2^{d}}}$. Similarly, there are a total of $\left(\mathrm{L} / 2^{d}\right) N^{d}$ locked sites which gives us the factor of $\left(e^{\mathrm{L} \beta \mu}\right)^{\frac{1}{2^{d}}}$. Next, it is seen that each of the $E$ bonds in $n_{c}$ produces $2 \times \frac{1}{2^{d}}$ bonds in the repeated pattern - the factor of two from the reflections orthogonal to the original bond - for a grand total of $e^{2 E \beta \kappa}$.

As for what remains, we denote by $\mathcal{G}_{n_{c}}(N)$ the graph that is obtained from the periodic extension of $n_{c}$ over $T_{N}$. (The bonds of $\mathcal{G}_{n_{c}}(N)$ are, of course, all of the nearest neighbor pairs in $\mathcal{G}_{n_{c}}(N)$.) Let $H_{N}^{n_{c}}$ denote the "uniform" Hamiltonian

$$
H_{N}^{n_{c}}=-\sum_{\langle i, j\rangle \in \mathcal{G}_{n_{c}}(N)} J\left(\sigma_{i}, \sigma_{j}\right)
$$

We will now estimate the trace of $e^{-\beta H_{N}^{n_{c}}}$. We claim that

$$
\operatorname{Tr} e^{-\beta H_{N}^{n_{c}}} \leq(q / s)^{N^{d-1}}\left[(s)^{\frac{\mathrm{L}}{2^{d}}}\right]^{N^{d}}
$$

from which the stated bound follows immediately.

To derive the inequality in the equation (3.7), recall the stipulation $J\left(\sigma_{i}, \sigma_{j}\right) \geq 0$; for an upper bound, this allows us to delete any interactions and perform a less restrictive trace. We will use this fact to prove the following (slightly) general result:

Let $\mathcal{G}$ denote any connected graph with $|\mathcal{G}|$ sites and let $H^{\mathcal{G}}$ denote a Hamiltonian that is of the form of the equation $(3.6)$ with $\mathcal{G}_{n_{c}}(N)$ replaced by $\mathcal{G}$. We claim that

$$
\operatorname{Tr} e^{-\beta H^{\mathcal{G}}} \leq q s^{|\mathcal{G}|-1}
$$

The proof follows by induction. The equation (3.8) is obviously true if $|\mathcal{G}|=2$. Suppose that it has been established for any connected graph of size $|\mathcal{G}|=k-1$. In adding the $k^{\text {th }}$ site, let us delete all but one of the bonds that attach the new site to the old graph. Freezing the spin configuration on the old graph and performing the partial trace over the new spin, we obtain a multiplicative factor - that is clearly no larger than $s$ - times the weight of the frozen state according to the old Hamiltonian. Bounding the multiplicative factor above by $s$, the stated claim is established.

The equation (3.7) now follows from the observation that $\mathcal{G}_{n_{c}}(N)$ can have no more than $N^{d-1}$ components and applying the preceding claim to each component. The desired result is now established.

Proof of Theorem 3.1. Clearly, the main task will be to demonstrate that some- 
$A$ and $B$. We claim that at the value $\left(\beta^{*}, \mu^{*}\right)$ with $\beta^{*}$ the inverse temperature from hypothesis (i) and $\mu^{*}=-\left[D \kappa+\frac{\epsilon \log q}{\beta^{*}}\right]$, the inequality

$$
\left\langle\chi_{n_{c}}\right\rangle_{N, \beta}^{\kappa, \mu} \leq P^{-\frac{1}{2^{d}}}
$$

holds unless $n_{c}=A$ or $B$. Indeed, estimating $\mathcal{Z}_{N, \beta}^{\frac{1}{N^{d}}} \geq q^{\frac{1}{2}} e^{\frac{1}{2} \beta \mu}$, we write

$$
\left\langle\chi_{n_{c}}\right\rangle_{N, \beta}^{\kappa, \mu} \leq \frac{q^{\mathrm{u}} e^{\mathrm{u} \beta \mu} e^{\mathrm{l} \beta \mu} s^{\mathrm{l}}}{q^{\frac{1}{2}} e^{\frac{1}{2} \beta \mu}} e^{\frac{2 \mathrm{E} \beta \kappa}{2^{d}}}
$$

where $\mathrm{u}=\mathrm{U} / 2^{d}$ and $\mathrm{l}=\mathrm{L} / 2^{d}$ are the fractions of unencumbered and locked sites. Next we observe that

$$
\frac{1}{2} \mathrm{~L} \leq \mathrm{E} \leq \frac{d}{2} \mathrm{~L}
$$

The lower bound is trivial. As for the upper bound, notice that each site in $c$ is contained in $d$ bonds of $c$. From each locked site, let us place an outward pointing arrow along all the $d$ bonds of $c$ that are attached to the site. The E "bonds" of $n_{c}$ are just those (lattice) bonds that are double covered. There cannot be more than $\frac{d}{2}$ such doubles and this is the upper bound in the equation (3.11).

Thus, in the equation (3.10), we may replace $E$ with $\frac{d}{2} \mathrm{~L}$ if $\kappa \geq 0$ or $\frac{1}{2} \mathrm{~L}$ if $\kappa<0$. In short

$$
\left\langle\chi_{n_{c}}\right\rangle_{N, \beta}^{\kappa, \mu} \leq \frac{s^{\mathrm{l}} q^{\mathrm{u}} e^{\mathrm{u} \beta \mu} e^{\mathrm{l} \beta \mu} e^{\mathrm{lD \beta \kappa}}}{q^{\frac{1}{2}} e^{\frac{1}{2} \beta \mu}}
$$

Here $D$ was defined in the statement of the theorem.

Setting $\beta=\beta^{*}$, we may replace $s$ by $q^{\epsilon}$. For $\mu=\mu^{*}$ defined by the condition

$$
q^{\epsilon} e^{\beta^{*} \mu^{*}} e^{D \beta^{*} \kappa}=1
$$

This yields

$$
\left\langle\chi_{n_{c}}\right\rangle \leq q^{-\left(\frac{1}{2}-\mathfrak{u}\right)} e^{-\left(\frac{1}{2}-\mathfrak{u}\right) \beta^{*} \mu^{*}} \leq\left(q e^{\beta^{*} \mu^{*}}\right)^{-\frac{1}{2^{d}}},
$$

where the last inequality is the worst case scenario. Namely, $u$ differs from $\frac{1}{2}$ by an absence of a single particle. Finally, we use the equation (3.13) and the condition (ii) to establish that $\left\langle\chi_{n_{c}}\left(\mathcal{T}_{N}\right)\right\rangle$ is indeed small.

Thus, if $P$ is sufficiently large, the cubes bearing $A$ or $B$ dominate and contours separating $A$ and $B$ regions are suppressed. The existence of two distinct staggered phases $A$ and $B$ is established by the same argument as in the proof of Theorem 2.1 (cf. equations (2.28) and (2.29)). 


\section{Bond AGgregation AND Site AGgREGAtion}

In this final section, we treat the bond and site aggregation transitions that were advertised in the introduction. Both of these transitions are reminiscent of the transition in $R_{I I}$ that was analyzed in Theorem 2.1 - indeed in the case of site aggregation, what we will prove is exactly the generalization of Theorem 2.1 part (ii). In both the bond and site problems, we will require very little: in each case, we will make some mild assumptions concerning the low energy behavior of the uniform system. These assumptions are satisfied in all the familiar spin models e.g. the $q$-state ferromagnets in the first example following the statement of Theorem 3.1. For the bond-dilute models we will require large $q$ (i.e. the phenomenon is entropy driven) but, of course, for the site diluted case this is not necessary.

Throughout this section, the uniform Hamiltonian will take the form of the equation $(3.1)$ with weights $(w(1), \ldots, w(q))$ that sum to $q$. The site-diluted models will therefore be exactly of the form in the equation (3.2) and the bond-diluted models will be of the form in the equation (1.2a) with spin couplings as described in the equation (3.1) and

$$
\lambda_{i, j}= \begin{cases}\lambda & \text { if } i \text { and } j \text { are neighbors } \\ 0 & \text { otherwise }\end{cases}
$$

For the bond-dilute case, let us formulate our conditions about the Hamiltonian $H_{u}$ from the equation (3.1). Recall the definition of $s(\beta)$ (c.f. the equation (3.4)) and let $\zeta(\beta)$ denote the partition function per site:

$$
\zeta(\beta)=\lim _{N \rightarrow \infty}\left(\mathcal{Z}_{N, \beta}\right)^{\frac{1}{N^{d}}}
$$

We will suppose that there is a $\beta \leq \infty$ and positive numbers $\epsilon$ and $\epsilon^{\prime}$ with $\epsilon+\epsilon^{\prime}<$ $\frac{1}{d 2^{d-1}}$ such that

$$
s(\beta) \leq q^{\epsilon}
$$

and

$$
\zeta(\beta) \geq q^{-\epsilon^{\prime}}
$$

If the above holds at $\beta=\infty$, then it also holds for $\beta$ sufficiently large.

We now state

Theorem 4.1. Consider the bond-diluted Hamiltonian as described above and suppose that $\beta$ is such that the conditions (4.3) hold. Then there is a value of bond "chemical potential" $\lambda=\lambda(\beta)$ such that two distinct phases $\langle-\rangle_{\beta, \lambda}^{\emptyset}$ and $\langle-\rangle_{\beta, \lambda}^{F}$ co-

exist. The state $\langle-\rangle_{\beta, \lambda}^{\emptyset}$ is characterized by a low density of bonds while in $\langle-\rangle_{\beta, \lambda}^{F}$, bonds are occupied with high probability.

Proof. We will show that if $q$ is large enough, then at inverse temperature $\beta$, along the entire isotherm, the only behaviors that are observed with any appreciable probability on cubes $c \in \mathcal{T}_{N}$ are "full" and "empty".

We start by noting that the considered bond-diluted Hamiltonian on a $d$-dimensional torus $\mathcal{T}_{N}$ is RP.

Let $m_{c} \equiv\left\{n_{i, j} \mid i, j \in c\right\}$ be a bond pattern on a cube $c$. The principal cases, $m_{c}=F$ (full) and $m_{c}=\emptyset$ (empty) give rise to constrained partition functions $\mathcal{Z}_{F}$ and $\mathcal{Z}_{\emptyset}$ that satisfy 
and

$$
\mathcal{Z}_{F}^{\frac{1}{N^{d}}} \gtrsim \zeta(\beta) e^{d \beta \lambda} \geq q^{-\epsilon^{\prime}} e^{2 d \beta \lambda}
$$

Let $\mathrm{B}$ denote the number of bonds in the pattern $m_{c}$, $\mathrm{U}$ denote the number of sites in $c$ that do not belong to any bond of $m_{c}$ and, finally, let $\mathrm{L}=2^{d}-\mathrm{U}$. By the chessboard estimate and the argument in Lemma 3.2 leading to the bound (3.8), it is not hard to see that

$$
\left\langle\chi_{m_{c}}\right\rangle_{N, \beta}^{\lambda} \leq \frac{\left(q^{\mathrm{U}} e^{2 \mathrm{~B} \beta \lambda} s^{\mathrm{L}}\right)^{\frac{1}{2^{d}}}}{\left[q^{N^{d}}+\left(q^{-\epsilon^{\prime}} e^{d \beta \lambda}\right)^{N^{d}}\right]^{\frac{1}{N^{d}}}},
$$

where $\chi_{m_{c}}$ is the indicator for the event $m_{c}$ and $\langle-\rangle_{N, \beta}^{\lambda}$ is the Gibbs state on $\mathcal{T}_{N}$ for the considered bond-diluted Hamiltonian. We write $\mathcal{R} \equiv q^{-\epsilon^{\prime}} e^{d \beta \lambda} / q$ so that, as $\lambda \rightarrow \pm \infty$, the empty/full configurations dominate. Writing the terms in the numerator and denominator in terms of $q$ and $\mathcal{R}$, we obtain

$$
\left\langle\chi_{m_{c}}\right\rangle_{N, \beta}^{\lambda} \leq \frac{\left(q^{\epsilon^{\prime} \frac{2 \mathrm{~B}}{d}} q^{\epsilon \mathrm{L}} q^{\left(\frac{2 \mathrm{~B}}{d}+\mathrm{U}\right)}\right)^{\frac{1}{2^{d}}}}{q} \frac{\left(\mathcal{R}^{\frac{2 \mathrm{~B}}{d}}\right)^{\frac{1}{2^{d}}}}{\left(1+\mathcal{R}^{N^{d}}\right)^{\frac{1}{N^{d}}}}
$$

Since $\mathrm{B}<d 2^{d-1}$, the factor involving $\mathcal{R}$ is uniformly bounded by one. We may also, with certain inefficiency, replace the coefficients of $\epsilon$ and $\epsilon^{\prime}$ by unity. We now claim that for $\mathrm{U} \neq 0$ or $2^{d}$, one has $\frac{2 \mathrm{~B}}{d}+\mathrm{U}<2^{d}$. This would easily follow from the (strict) inequality that $\mathrm{B}<\frac{d}{2} \mathrm{~L}$ unless $\mathrm{L}=0$ or $2^{d}$. However, supposing that $\mathrm{B}=\frac{d}{2} \mathrm{~L}$ and $\mathrm{L}>0$, then it must be the case that each site attached to any bond is in fact attached to all $d$ possible bonds in $c$ emanating from it. But this would, necessarily, cover all of $c$.

Thus, for the cases of relevance, we may claim

$$
\frac{2 \mathrm{~B}}{d}+\mathrm{u} \leq 2^{d}-\frac{2}{d}
$$

and hence

$$
\left\langle\chi_{m_{c}}\right\rangle_{N, \beta}^{\lambda} \leq q^{\left(\epsilon+\epsilon^{\prime}-\frac{1}{d 2^{d-1}}\right)}
$$

Thus, for $\epsilon$ and $\epsilon^{\prime}$ as stated and $q$ sufficiently large, using the routine contour argument, we may plug directly into Lemma 2.4 and the result is established.

In the site diluted case, we will need no assumption concerning the value of $s(\beta)$ - the obvious bound $s(\beta)<q^{2}$ is sufficient. However, we will require that the low temperature behavior of the uniform system is not too badly frustrated. Namely, we will suppose that there is a constant $\Delta<\frac{\kappa}{d 2^{d-1}}$ and a $\beta^{*}<\infty$ such that

$$
\zeta(\beta) \geq e^{-\beta \Delta}
$$

for all $\beta>\beta^{*}$. We reemphasize that in all of the usual problems, the condition 
Theorem 4.2. Consider the site-diluted Hamiltonians with $\kappa>0$ and suppose that the condition (4.10) holds. Then there is a value $\mu^{*}(\beta)$ at which two phases, "full" and "empty", coexist.

Proof. It is sufficient, by the previous arguments to establish that $\left\langle\chi_{n_{c}}\right\rangle_{N, \beta}^{J, \mu, \kappa} \ll 1$, unless $n_{c}$ is full or empty, uniformly in $\mu$. For $\kappa>0$ this will be shown for all $\beta$ sufficiently large. By the standard estimates, we may bound

$$
\left\langle\chi_{n_{c}}\right\rangle_{N, \beta}^{J, \mu, \kappa} \leq \frac{\left(q^{\mathrm{U}} e^{\mathrm{U} \beta \mu} e^{2 \mathrm{E} \beta \kappa} s^{\mathrm{L}}\right)^{\frac{1}{2^{d}}}}{\left[1+\left(e^{\beta \mu} e^{d \beta \kappa} e^{-\Delta \beta}\right)^{N^{d}}\right]^{\frac{1}{N^{d}}}} .
$$

After some manipulations on the right hand side,

$$
\left\langle\chi_{n_{c}}\right\rangle_{N, \beta}^{J, \mu, \kappa} \leq e^{(\mathrm{l}+\mathrm{u}) \Delta \beta} q^{\mathfrak{u}} s^{\mathrm{l}} e^{-[d(\mathrm{~L}+\mathrm{U})-2 \mathrm{E}] \beta \kappa \frac{1}{2^{d}}} \frac{e^{(\mathrm{l}+\mathfrak{u})[\beta \mu+d \beta \kappa]}}{\left[1+\left(e^{\beta \mu} e^{d \beta \kappa} e^{-\Delta \beta}\right)^{N^{d}}\right]^{\frac{1}{N^{d}}}} .
$$

We will immediately write $l+u \leq 1$, which allows us to get rid of the fraction and to replace the first term by $e^{\Delta \beta}$. Next, we claim that for $n_{c}$ not full or empty, $d(\mathrm{~L}+\mathrm{U})-\mathrm{E} \geq \frac{2}{d}$. Indeed, this is basically the same argument that was used in Theorem 4.1. Namely, if $\mathrm{E}=\mathrm{L}=0$, we must have $\mathrm{U} \geq 1$. Otherwise, for $\mathrm{L}<2^{d}$ we have $2 \mathrm{E}<d \mathrm{~L}$, i.e. $2 \mathrm{E} \leq d \mathrm{~L}-1$. Putting these facts together, we arrive at

$$
\left\langle\chi_{n_{c}}\right\rangle_{N, \beta}^{J, \mu, \kappa} \leq q^{\mathrm{u}} s^{\mathrm{l}} e^{-\beta\left[\frac{\kappa}{d 2^{d-1}}-\Delta\right]} \rightarrow 0 \text { as } \beta \rightarrow \infty
$$

If the equation (4.13) is taken in conjunction with the previous arguments, then for all $\beta$ sufficiently large, phase coexistence at some $\mu^{*}(\beta)$ is established.

The authors would like to thank Roland Dobrushin for the tip that the $\infty>\mu \gg 1$ region of the site diluted Potts models were accessible by these techniques. 


\section{REFERENCES}

[CKS] L. Chayes, R. Kotecký and S. B. Shlosman, Research in Progress.

[D]

$[\mathrm{DS}]$

$[\mathrm{EG}]$

$[\mathrm{F}]$

$[\mathrm{FL}]$

[FLIS I]

[FLIS III] J. Fröhlich, R. Israel, E. H. Lieb and B. Simon, Phase Transitions and Reflection Positivity. III, (In preparation).

[FSS] J. Fröhlich, B. Simon and T. Spencer, Infra-red Bounds, Phase Transitions and Continuous Symmetry Breaking, Comm. Math. Phys. 50 (1976), 79-95.

[HB] W. Hoston and A.N. Berker, Dimensionality Effects on the Multicritical Phase Diagrams of the Blume-Emery-Griffiths Model with Repulsive Biquadratic Couplings: Mean-field and Renormalization Group Studies, J. Appl. Phys. 70 (1991), 6101-6103.

[KS] R. Kotecký and S. B. Shlosman, First-Order Phase Transitions in Large Entropy Lattice Models, Comm. Math. Phys. 83 (1982), 493515.

[M] Martirosian, D. H., Translation invariant Gibbs states in q-state Potts model, Commun. Math. Phys. 105 (1986), 281-290.

[MS] R.A. Minlos and Y.A. Sinai, The Phenomenon of "Phase Separation" at Low Temperature in Some Lattice Gas Models I, Mat. Sb. Phys. 73 (1967), 375-488.

[NBRS] B. Nienhuis, A.N. Berker, E.K. Riedel and M. Schick, First- and Second-Order Phase Transitions in Potts Models RenormalizationGroup Solution, Phys. Rev. Lett. 43 (1979), 737-740.

[RL] L.K. Runels and J.L. Lebowitz, Phase Transitions of a Multicomponent Widom-Rowlinson Model, Jour. Math. Phys. 15 (1974), 17121717.

[S] S. B. Shlosman, The Method of Reflection Positivity in the Mathematical Theory of First-Order Phase Transitions, Russian Math. Surveys 
[St] R.B. Stinchcombe, Dilute Magnetism, Phase Transitions and Critical Phenomena Vol.7, Edited by C. Domb and J.L. Lebowitz, Academic Press Inc., London, 1983.

[SW] S. Sarbach and F. Y. Wu, Z Phys. B 44 (1981), 309.

[SM] I. Syozi and S. Miyazima, Prog. Theor. Phys. 36 (1966), 1803.

[ST] B.W. Southern and M.F. Thorpe, J. Phys. C 12 (1979), 5351.

[Z] M. Zahradník, An Alternate Version of Pirogov-Sinai Theory, Commun. Math. Phys. 93 (1984), 559-581. 
Lincoln Chayes

Department of Mathematics

UNIVERSITY OF CALIFORNIA

Los ANGeles, CAlifornia 90024

E-mail address: 1chayes@math.ucla.edu

\section{ROMAN KOTECKÝ}

Center for Theoretical Study, Charles University,

TÁboritská 23, 13087 Praha 3, Czech Republic

AND

Department of Theoretical Physics, Charles University,

V HolešovičKách 2, 18000 Praha 8, Czech Republic

E-mail address: kotecky@cspuni12.bitnet

SENYA SHLOSMAN

DEPARTMENT OF MATHEMATICS

UNIVERSITY OF CALIFORNIA

IRVine, CALIFORnia 92717

E-mail address: shlosman@math.uci.edu 